\title{
THE MUSCLES TREASURY SURVEY. I. MOTIVATION AND OVERVIEW*
}

\author{
Kevin France $^{1}$, R. O. Parke Loyd ${ }^{1}$, Allison Youngblood ${ }^{1}$, Alexander Brown ${ }^{2}$, P. Christian Schneider ${ }^{3}$, \\ Suzanne L. Hawley ${ }^{4}$, Cynthia S. Froning ${ }^{5}$, JefFrey L. Linsky ${ }^{6}$, Aki Roberge ${ }^{7}$, Andrea P. Buccino ${ }^{8}$, \\ James R. A. Davenport ${ }^{9,19}$, Juan M. Fontenla ${ }^{10}$, Lisa Kaltenegger $^{11}$, Adam F. Kowalski ${ }^{12}$, Pablo J. D. Mauas ${ }^{8}$, \\ Yamila Miguel ${ }^{13}$, Seth Redfield ${ }^{14}$, Sarah Rugheimer ${ }^{15}$, Feng Tian ${ }^{16}$, Mariela C. Vieytes ${ }^{17}$, \\ LuCianne M. Walkowicz ${ }^{18}$, and Kolby L. Weisenburger ${ }^{4}$ \\ ${ }^{1}$ Laboratory for Atmospheric and Space Physics, University of Colorado, 600 UCB, Boulder, CO 80309, USA; kevin.france@ @olorado.edu \\ ${ }^{2}$ Center for Astrophysics and Space Astronomy, University of Colorado, 389 UCB, Boulder, CO 80309, USA \\ ${ }^{3}$ European Space Research and Technology Centre (ESA/ESTEC), Keplerlaan 1, 2201 AZ Noordwijk, The Netherlands \\ ${ }_{5}^{4}$ Department of Astronomy, University of Washington, Box 351580, Seattle, WA 98195, USA \\ 5 Department of Astronomy, C1400, University of Texas at Austin, Austin, TX 78712, USA \\ 6 JILA, University of Colorado and NIST, 440 UCB, Boulder, CO 80309, USA \\ ${ }^{7}$ Exoplanets and Stellar Astrophysics Laboratory, NASA Goddard Space Flight Center, Greenbelt, MD 20771, USA \\ ${ }^{8}$ Instituto de Astronomía y Física del Espacio (UBA-CONICET) and Departamento de Física (UBA), CC.67, suc. 28, 1428, Buenos Aires, Argentina \\ ${ }^{9}$ Department of Physics \& Astronomy, Western Washington University, Bellingham, WA 98225, USA \\ ${ }^{10}$ NorthWest Research Associates, 3380 Mitchell Lane, Boulder, CO 80301-2245, USA \\ ${ }^{11}$ Carl Sagan Institute, Cornell University, Ithaca, 14850, NY, USA
${ }^{12}$ Department of Astronomy, University of Maryland, College Park, MD 20742, USA \\ ${ }^{13}$ Laboratoire Lagrange, Universite de Nice-Sophia Antipolis, Observatoire de la Cote d'Azur, CNRS, \\ Blvd de l'Observatoire, CS 34229, F-06304 Nice cedex 4, France \\ ${ }^{14}$ Astronomy Department and Van Vleck Observatory, Wesleyan University, Middletown, CT 06459-0123, USA \\ ${ }^{15}$ Department of Earth and Environmental Sciences, Irvine Building, University of St. Andrews, St. Andrews KY16 9AL, UK \\ ${ }^{16}$ Ministry of Education Key Laboratory for Earth System Modeling, Center for Earth System Science, Tsinghua University, Beijing 100084 , China \\ ${ }^{17}$ Instituto de Astronomía y Física del Espacio (UBA-CONICET) and UNTREF, CC.67, suc. 28, 1428, Buenos Aires, Argentina \\ ${ }_{18}$ The Adler Planetarium, 1300 S Lakeshore Drive, Chicago, IL 60605, USA \\ Received 2015 December 17; accepted 2016 February 19; published 2016 March 22
}

\begin{abstract}
Ground- and space-based planet searches employing radial velocity techniques and transit photometry have detected thousands of planet-hosting stars in the Milky Way. With so many planets discovered, the next step toward identifying potentially habitable planets is atmospheric characterization. While the Sun-Earth system provides a good framework for understanding the atmospheric chemistry of Earth-like planets around solar-type stars, the observational and theoretical constraints on the atmospheres of rocky planets in the habitable zones (HZs) around low-mass stars ( $\mathrm{K}$ and $\mathrm{M}$ dwarfs) are relatively few. The chemistry of these atmospheres is controlled by the shape and absolute flux of the stellar spectral energy distribution (SED), however, flux distributions of relatively inactive low-mass stars are poorly understood at present. To address this issue, we have executed a panchromatic (X-ray to mid-IR) study of the SEDs of 11 nearby planet-hosting stars, the Measurements of the Ultraviolet Spectral Characteristics of Low-mass Exoplanetary Systems (MUSCLES) Treasury Survey. The MUSCLES program consists visible observations from Hubble and ground-based observatories. Infrared and astrophysically inaccessible wavelengths (EUV and $\mathrm{Ly} \alpha$ ) are reconstructed using stellar model spectra to fill in gaps in the observational data. In this overview and the companion papers describing the MUSCLES survey, we show that energetic radiation (X-ray and ultraviolet) is present from magnetically active stellar atmospheres at all times for stars as late as M6. The emission line luminosities of $\mathrm{C}_{\mathrm{IV}}$ and $\mathrm{Mg}$ II are strongly correlated with band-integrated luminosities and we present empirical relations that can be used to estimate broadband FUV and XUV ( $\equiv$ X-ray + EUV) fluxes from individual stellar emission line measurements. We find that while the slope of the SED, FUV/NUV, increases by approximately two orders of magnitude form early $\mathrm{K}$ to late M dwarfs $(\approx 0.01-1)$, the absolute FUV and XUV flux levels at their corresponding HZ distances are constant to within factors of a few, spanning the range $10-70 \mathrm{erg} \mathrm{cm}^{-2} \mathrm{~s}^{-1}$ in the HZ. Despite the lack of strong stellar activity indicators in their optical spectra, several of the $\mathrm{M}$ dwarfs in our sample show spectacular UV flare emission in their light curves. We present an example with flare/quiescent ultraviolet flux ratios of the order of 100:1 where the transition region energy output during the flare is comparable to the total quiescent luminosity of the star $E_{\text {flare }}(\mathrm{UV}) \sim 0.3 L_{*} \Delta t(\Delta t=1 \mathrm{~s})$. Finally, we interpret enhanced $L($ line $) / L_{\mathrm{Bol}}$ ratios for $\mathrm{C}$ Iv and $\mathrm{N} v$ as tentative observational evidence for the interaction of planets with large planetary mass-to-orbital distance ratios $\left(M_{\text {plan }} / a_{\text {plan }}\right)$ with the transition regions of their host stars.
\end{abstract}

Key words: planetary systems - stars: activity - stars: low-mass

\section{INTRODUCTION}

\footnotetext{
* Based on observations made with the NASA/ESA Hubble Space Telescope, obtained from the data archive at the Space Telescope Science Institute. STScI is operated by the Association of Universities for Research in Astronomy, Inc. under NASA contract NAS 5-26555.

${ }^{19}$ NSF Astronomy and Astrophysics Postdoctoral Fellow.
}

The Kepler mission and ground-based planet searches have detected thousands of exoplanets within the Milky Way and have demonstrated that approximately one in six mainsequence FGK stars hosts an Earth-size planet (with periods 
up to 85 days; Fressin et al. 2013). One of the highest priorities for astronomy in the coming decades is the characterization of the atmospheres, and possibly the surfaces, of Earth-size planets in the habitable zones (HZs, where liquid water may exist on terrestrial planet surfaces) around nearby stars. An intermediate step toward the discovery of life on these worlds is the measurement of atmospheric gases that may indicate the presence of biological activity. These gases are often referred to as biomarkers or biosignatures.

However, the planetary effective surface temperature alone is insufficient to accurately interpret biosignature gases when they are observed in the coming decades. The dominant energy input and chemistry driver for these atmospheres is the stellar spectral energy distribution (SED). The ultraviolet (UV) stellar spectrum which drives and regulates the upper atmospheric heating and chemistry on Earth-like planets is critical to the definition and interpretation of biosignature gases (e.g., Seager et al. 2013), and may even produce false-positives in our search for biologic activity (Hu et al. 2012; Domagal-Goldman et al. 2014; Tian et al. 2014)

The nearest potentially habitable planet is likely around an M dwarf at $d<3$ pc (Dressing \& Charbonneau 2015), the nearest known Earth-size planet orbits an $\mathrm{M}$ dwarf (GJ 1132b, $R_{p}=1.2 R_{\oplus}, d=12 \mathrm{pc}$; Berta-Thompson et al. 2015), and the nearest known super-Earth-mass planets in HZs orbit $\mathrm{M}$ and $\mathrm{K}$ dwarfs, making planetary systems around low-mass stars prime targets for spectroscopic biomarker searches (see also Cowan et al. 2015). The low ratio of stellar-to-planetary mass more readily permits the detection of lower-mass planets using the primary detection techniques (radial velocity and transits). Moreover, the $\mathrm{HZ}$ around a star moves inward with decreasing stellar luminosity. These factors make potentially habitable planets easier to detect around $\mathrm{M}$ and $\mathrm{K}$ dwarfs. The importance of $\mathrm{M}$ dwarf exoplanetary systems is underscored by recent Kepler results and radial velocity measurements showing that between $\sim 10 \%$ and $50 \%$ of M dwarfs host Earthsize planets $\left(0.5-1.4 R_{\oplus}\right)$ in their HZs (Bonfils et al. 2013; Kopparapu 2013; Dressing \& Charbonneau 2015). Furthermore, approximately $70 \%$ of the stars in the Milky Way are M dwarfs, and so rocky planets around low-mass stars likely dominate the planet distribution of the Galaxy. Theoretical work has shown that planets around $\mathrm{M}$ dwarfs could be habitable despite their phase-locked orbits (Joshi 2003) and dynamic modeling of transiting systems reveals that most systems permit stable orbits of Earth mass planets in the $\mathrm{HZ}$ long enough for the development of life, i.e., $\gtrsim 1.7 \mathrm{Gyr}$ (Jones \& Sleep 2010).

$\mathrm{M}$ and $\mathrm{K}$ dwarfs show significantly larger temporal variability and a larger fraction of their bolometric luminosity at UV wavelengths than solar-type stars (France et al. 2013), yet their actual spectral and temporal behavior is not well studied, except for a few young ( $<1 \mathrm{Gyr}$ ), active flare stars. The paucity of UV spectra of low-mass stars and our current inability to accurately model the UV spectrum of a particular M or $\mathrm{K}$ dwarf without a direct observation limits our ability to reliably predict possible atmospheric biomarkers. Without the stellar UV spectrum, we cannot produce realistic synthetic spectra of Earth-like planets in these systems, a necessary step for interpreting biomarker gases and their potential to diagnose habitability. Therefore, our quest to observe and characterize biosignatures on rocky planets must consider the star-planet

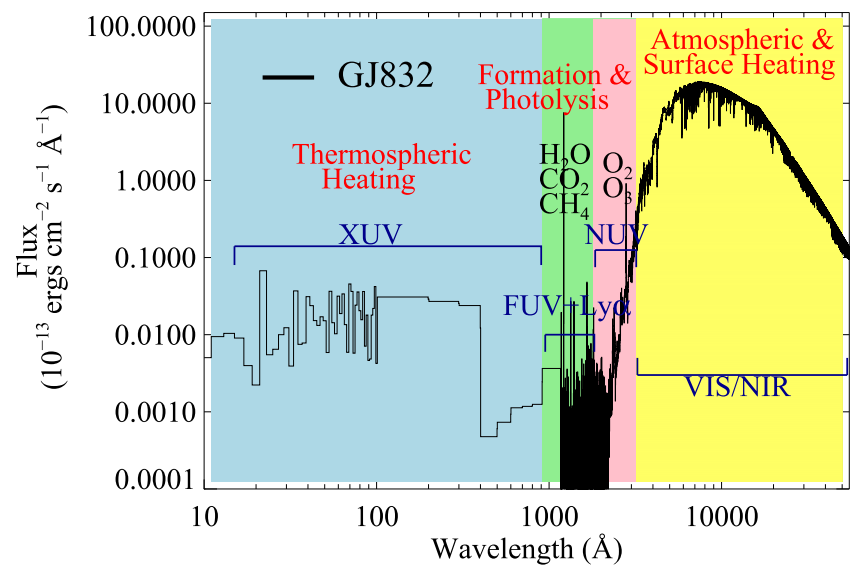

Figure 1. Panchromatic spectrum of GJ 832, illustrating the influence of each spectral bandpass on an Earth-like planet orbiting this star. GJ 832 has a superEarth mass planet located in the HZ (Wittenmyer et al. 2014).

system as a whole, including the interaction between the stellar irradiance and the exoplanetary atmosphere.

\subsection{High-energy Spectra as Photochemical Atmospheric Model Inputs}

FUV and NUV Irradiance: Photochemistry and Biosignatures-Spectral observations of $\mathrm{O}_{2}, \mathrm{O}_{3}, \mathrm{CH}_{4}$, and $\mathrm{CO}_{2}$ are expected to be the most important signatures of biological activity on planets with Earth-like atmospheres (Des Marais et al. 2002; Kaltenegger et al. 2007; Seager et al. 2009). The chemistry of these molecules in the atmosphere of an Earth-like planet depends sensitively on the strength and shape of the UV spectrum of the host star (Segura et al. 2005). $\mathrm{H}_{2} \mathrm{O}, \mathrm{CH}_{4}$, and $\mathrm{CO}_{2}$ are sensitive to FUV radiation $(912-1700 \AA)$, in particular, the bright HI Ly $\alpha$ line, while the atmospheric oxygen chemistry is driven by a combination of FUV and NUV (1700-3200 ^) radiation (Figure 1).

The photolysis (photodissociation) of $\mathrm{CO}_{2}$ and $\mathrm{H}_{2} \mathrm{O}$ by $\mathrm{Ly} \alpha$ and other bright stellar chromospheric and transition region emission lines (e.g., $\mathrm{C}_{\text {II }} \lambda 1335 \AA$ and $\mathrm{C}$ IV $\lambda 1550 \AA$ ) can produce a buildup of $\mathrm{O}_{2}$ on planets illuminated by strong FUV radiation fields. Once a substantial $\mathrm{O}_{2}$ atmosphere is present, $\mathrm{O}_{3}$ is primarily created through a multi-step reaction whereby $\mathrm{O}_{2}$ dissociation (by $1700-2400 \AA$ photons) is followed by the reaction $\mathrm{O}+\mathrm{O}_{2}+\xi \rightarrow \mathrm{O}_{3}+\xi$, where $\xi$ is a reaction partner required to balance energy conservation. $\mathrm{O}_{3}$ photolysis is then driven by NUV and blue optical photons. Therefore, on planets orbiting stars with strong FUV and weak NUV flux, a substantial $\mathrm{O}_{3}$ atmosphere may arise via photochemical processes alone (Segura et al. 2010; $\mathrm{Hu}$ et al. 2012; Gao et al. 2015; Harman et al. 2015).

This strong photochemical source of $\mathrm{O}_{3}$ may be detectable by future space observatories designed to carry out direct atmospheric spectroscopy of rocky planets (e.g., the HDST or LUVOIR mission concepts), and may be misinterpreted as evidence for biologic activity on these worlds. Therefore, characterization of the stellar FUV/NUV ratio is an essential complement to spectroscopy of exoplanet atmospheres to control for potential false-positive "biomarkers." Furthermore, it has been shown that the abundances of water and ozone, as well as the atmospheric equilibrium temperature, can respond to changes in the stellar flux on timescales ranging from minutes to years (Segura et al. 2010). Detailed knowledge of 
the absolute flux level and temporal behavior stellar spectrum is important for understanding the evolution of potentially habitable atmospheres.

$X$-ray and EUV Irradiance: Atmospheric Heating and Massloss - The ratios of X-ray to total luminosity of $\mathrm{M}$ dwarfs are orders of magnitude higher $(\gtrsim 10-100 \times)$ than those of the present day Sun (Poppenhaeger et al. 2010), and the smaller semimajor axes of the $\mathrm{HZ}$ around $\mathrm{M}$ dwarfs means that X-ray effects on $\mathrm{HZ}$ planets will likely be more important than on $\mathrm{HZ}$ planets orbiting solar-type stars (Cecchi-Pestellini et al. 2009). Soft X-ray heating of planetary atmospheres enhances evaporation and atmospheric escape (Scalo et al. 2007; Owen \& Jackson 2012), which may impact the long-term stability of an exoplanetary atmosphere. Recent works suggest that the influences of early evolution of low-mass stars and XUV heating could lead to a bi-modal distribution of water fractions on Earth mass planets in the HZ of $\mathrm{M}$ dwarfs (Tian \& Ida 2015). In order to model the atmosphere as a system, we require inputs for both heating (soft X-ray and EUV, see below) and photochemistry (FUV and NUV).

Extreme-UV (EUV; $100 \lesssim \lambda \lesssim 911 \AA$ ) photons from the central star are an important source of atmospheric heating and ionization on all types of extrasolar planets. For terrestrial atmospheres, increasing the EUV flux to levels estimated for the young Sun $(\approx 1 \mathrm{Gyr}$; Ayres 1997) can increase the temperature of the thermosphere by a factor of $\gtrsim 10$ (Tian et al. 2008), potentially causing significant and rapid atmospheric mass-loss. Ionization by EUV photons and the subsequent loss of atmospheric ions to stellar wind pick-up can also drive extensive atmospheric mass-loss on geologic timescales (e.g., Rahmati et al. 2014 and references therein). Estimates of the incident EUV flux are therefore important for evaluating the long-term stability of an $\mathrm{HZ}$ atmosphere; however, a direct measurement of the EUV irradiance from an exoplanetary host star is essentially impossible because interstellar hydrogen removes almost all of the stellar EUV flux for virtually all stars except the Sun. The stellar EUV energy budget contains contributions from both the transition region (Lyman continuum as well as helium and metal line emission in the 228-911 $\AA$ bandpass) and the corona. FUV emission lines (Ly $\alpha, \mathrm{C}$ IV, and $\mathrm{Si}$ IV) are required to estimate the contribution of the transition region to the EUV flux (Fontenla et al. 2011; Linsky et al. 2014), while X-ray data are necessary to constrain the contribution of the corona (e.g., Sanz-Forcada et al. 2011).

\subsection{Variability on Timescales of Minutes to Hours: Atmospheric Abundances}

An important measurement relating to the habitability of extrasolar planets is the time variability of the energetic incident radiation. While most of the quiescent UV emission from $M$ dwarfs comes from emission lines, continuum emission can become the dominant UV luminosity source during flares (Kowalski et al. 2010). The relative UV emission line strengths also vary during flares (e.g., Hawley et al. 2003; Osten et al. 2005; Loyd \& France 2014). Thus, molecular species in the atmospheres of HZ planets will be "selectively pumped" during quiescent periods; only species that have spectral coincidences with stellar emission lines will be subject to large energy input from the host star. However, during flares with strong continuum emission, the relative excitation and dissociation rates relative to quiescent periods could change radically. Therefore, temporally and spectrally resolved observations are essential for understanding the impact of time variability on $\mathrm{HZ}$ planetary atmospheres. The amplitude and frequency of flare activity on older M-star exoplanetary hosts is completely unexplored, although GALEX NUV imaging observations suggest that flares may significantly alter the steady-state chemistry in the atmospheres of planets in the HZ (Welsh et al. 2006).

Impulsive UV events are also signposts for energetic flares associated with large ejections of energetic particles. Segura et al. (2010) have shown that energetic particle deposition into the atmosphere of an Earth-like planet without a magnetic field during a large $\mathrm{M}$ dwarf flare can lead to significant atmospheric $\mathrm{O}_{3}$ depletion ( $>90 \%$ for the most extreme flares). Buccino et al. (2007) also studied the impact of a series of lower intensity flares from highly active stars. These events could alter the atmospheric chemistry and increase the penetration depth of UV photons that are damaging to surface life. The impact of a single flare may be detrimental to the development and maintenance of life, but the potentially far more significant impact of persistent flare events has not been studied because the temporal behavior of UV flares is unexplored outside of a few extreme M dwarf flare stars (e.g., AD Leo, EV Lac, and AU Mic; e.g., Hawley et al. 1991; Robinson et al. 2001; Osten et al. 2005).

\subsection{The MUSCLES Treasury Survey: An Energetic Radiation Survey of Exoplanetary Hosts}

With the previously described motivation in mind, the question that arises is "what are the shapes and absolute flux levels of the UV stellar SEDs incident on these planetary systems?" At present, GJ 832 is the only M dwarf for which a semi-empirical atmosphere model has been built and vetted by direct comparison with spectroscopic observations at optical, NUV, FUV, and X-ray wavelengths (Fontenla et al. 2016). There are no other stellar atmosphere models for $\mathrm{M}$ dwarfs that treat the chromosphere, transition region, and corona in a selfconsistent manner, and none that can produce synthetic spectra for the important X-ray and ultraviolet bandpasses (5-3000 ̊).

Several approaches have been taken in the literature, including assuming that the star has no UV emission (essentially, no magnetically active upper atmosphere; Segura et al. 2005; Kaltenegger et al. 2011), assuming the extreme flare environment of a star like AD Leo (Segura et al. 2010; Wordsworth et al. 2010), or using low signal-to-noise ratio (S/ $\mathrm{N})$ observations of the few flaring $\mathrm{M}$ dwarfs that could be observed by IUE (Segura et al. 2005; Buccino et al. 2007). Previously available archival data are insufficient for an accurate quantitative analysis of the radiation fields incident on potentially habitable planets orbiting $\mathrm{M}$ dwarfs. Lowsensitivity and contamination by geocoronal $\operatorname{Ly} \alpha$ emission make IUE observations insufficient for this work (see example in Section 2). The lack of observational constraints from the UV spectra of M dwarfs will have a major impact on how we judge whether or not the planets in these systems are actually inhabited. While the need for panchromatic data has been realized for Sun-like stars (e.g., Sun in Time; Ribas et al. 2005), M dwarfs have received less attention (see also Guinan \& Engle 2009), despite the fact that these systems dominate the planet statistics of the Galaxy.

To address the above question, we have carried out the first panchromatic survey of $\mathrm{M}$ and $\mathrm{K}$ dwarf exoplanet host stars in 
the solar neighborhood $(d \lesssim 20 \mathrm{pc})$. We refer to this program as the Measurements of the Ultraviolet Spectral Characteristics of Low-mass Exoplanetary Systems (MUSCLES) Treasury Survey, a coordinated X-ray to NIR observational effort to provide the exoplanet community with empirically derived panchromatic irradiance spectra for the study of all of the types of exoplanets orbiting these stars. MUSCLES is largely based on a Hubble Space Telescope (HST) Cycle 22 treasury program and makes use of smaller guest observing programs on HST, XMM-Newton, Chandra, and several ground-based observatories.

Our survey provides a database of the chromospheric, transition region, and coronal properties of low-mass stars hosting exoplanets, providing high-quality input for models of both Jovian and Earth-like planets as vast numbers of these systems are discovered and characterized in the next decade with missions such as WFIRST, Plato, TESS, and the James Webb Space Telescope (JWST). While the long-term evolution of the broadband UV luminosity function of $\mathrm{M}$ dwarfs can be constrained with large photometric samples from GALEX (e.g., Shkolnik \& Barman 2014), a further uncertainty in the temporal behavior of low-mass exoplanet host stars is the variability on timescales of years (the stellar equivalent of the solar cycle). Given the limitations on space observatories, particularly as it is likely that we will be in the post-HST and post-Chandra era within the next 5-10 years, it is critically important to identify a set of visible-wavelength tracers (e.g., Gomes da Silva et al. 2011) that can be used to quantify the longer-term (years-to-decades) UV variability of these stars. The MUSCLES data set will enable us to derive empirical relations between the optical, UV, and X-ray fluxes (e.g., FUV luminosity versus $\mathrm{Ca}$ II and $\mathrm{H} \alpha$ profiles), as well as their relative behavior during flares, supporting long-term, groundbased programs to study the time evolution of the energetic radiation environment.

This paper provides an overview of the motivation for and design of the MUSCLES Treasury Survey, as well as some initial quantitative results. A detailed example of the need for $H S T$ to carry out this work is given is Section 2. Section 3 describes the MUSCLES target list and the description of the observing modes used in the program. Section 4 presents the evolution of the broadband SED with stellar effective temperature and $\mathrm{HZ}$ distance, provides scaling relations to estimate the broadband luminosity from individual spectral line measurements, and presents a first look at the intense highenergy flare behavior of these otherwise inactive stars. Section 5 explores the interaction of the planets and host stars in these systems, and compares the UV flux measurements with predictions from coronal models. Section 6 presents a summary of the important results from this paper.

Details on all of the aspects of the data analysis and scientific results from the program can be found in the companion papers from the MUSCLES team. Youngblood et al. (2016) present an analysis of the essential reconstruction of the intrinsic Ly $\alpha$ stellar emission lines and the calculations of the EUV irradiance ("Paper II"). Loyd et al. (2016), "Paper III," describe the creation of the panchromatic SEDs, quantification of the SEDs for exoplanet atmospheric modeling, and a description of how to download the data in machine-readable format from the Milkulski Archive for Space Telescopes. ${ }^{20}$ Fontenla et al.

\footnotetext{
$\overline{{ }^{20} \text { https://archive.stsci.edu/prepds/muscles/ }}$
}

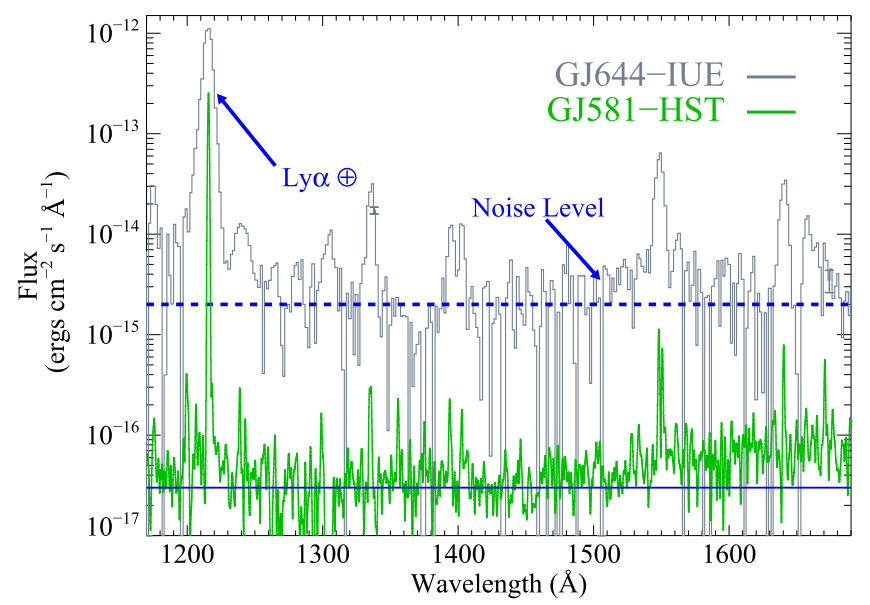

Figure 2. Comparison of the IUE spectrum of the M3V star GJ 644 and the weakly active M3V exoplanet host star GJ 581 (France et al. 2013). The IUE spectrum is severely contaminated by geocoronal Ly $\alpha$ and $\mathrm{O}_{\mathrm{I}} \lambda 1304$ emission and lacks the spectral resolution needed to permit an intrinsic profile reconstruction (see, e.g., Wood et al. 2005b). The blue dashed line represents the instrumental background level in IUE FUV observations $\left(F_{\lambda} \approx 2 \times 10^{-15} \mathrm{erg} \mathrm{cm}^{-2} \mathrm{~s}^{-1} \AA^{-1}\right)$. Given the geocoronal emission and instrumental background levels, we estimate that approximately $80 \%$ of the total 1160-1690 A FUV flux in the GJ 644 spectrum is contributed by nonstellar sources. Higher sensitivity, higher spectral resolution observations are essential for accurately measuring the energetic radiation environment in the $\mathrm{HZ}$ of low-mass stars.

(2016) present semi-empirical modeling of $\mathrm{M}$ dwarf atmospheres based in part on MUSCLES observations. Detailed descriptions of the flare properties of the MUSCLES sources and a comparison of the UV and X-ray emission from these stars with contemporaneous ground-based photometry and spectroscopy are presented in papers by R. O. P. Loyd (2016, in preparation) and A. Youngblood (2016, in preparation), respectively, and J. Linsky et al. (2016, in preparation) describe the kinematics of the stellar atmospheres derived from the UV emission lines in the HST data.

\section{MOTIVATION: THE IMPORTANCE OF HIGH- SENSITIVITY, SPECTRALLY RESOLVED DATA FOR ATMOSPHERIC MODELING}

As mentioned above, our observational and theoretical understanding of the upper atmospheres of "weakly active" M dwarfs (Walkowicz \& Hawley 2009) is insufficient for a deterministic prediction of the flux in the $\mathrm{HZ}$ around an $\mathrm{M}$ dwarf host star. GALEX data provide a statistical picture of the evolution of the UV luminosity of M dwarfs (Shkolnik \& Barman 2014), but do not include spectral information on the specific intensity (important for accurate photoexcitation and photolysis rates of key atmospheric species), coverage of the brightest FUV and NUV spectral lines (Ly $\alpha$ and $\mathrm{Mg}$ II, respectively), information about the flare or stellar cycle state of the star, or the connection to the X-ray and optical fluxes of the stars. Shkolnik et al. (2014) provided scaling relations to calculate the $\operatorname{Ly} \alpha$ and $\mathrm{Mg}$ II fluxes from GALEX broadband fluxes when HST spectroscopy is not available.

To demonstrate the importance of using high-quality UV observations from $H S T$, in Figure 2 we compare the observed UV flux of GJ 581 with another M3 (spectral types for GJ 581 range from M2.5 to M5 in the literature) stellar spectrum taken from the $I U E$ archive (the M3V binary star GJ 644, scaled to the distance of GJ 581). One might consider using GJ 
Table 1

MUSCLES Treasury Survey-Target List

\begin{tabular}{|c|c|c|c|c|c|c|c|c|}
\hline Star & $\begin{array}{l}\text { Distance } \\
(\mathrm{pc})\end{array}$ & Type & $\begin{array}{l}T_{\text {eff }} \\
(\mathrm{K})\end{array}$ & $\begin{array}{c}P_{\text {rot }} \\
\text { (days) }\end{array}$ & $\begin{array}{c}\left\langle r_{\mathrm{HZ}}\right\rangle^{\mathrm{a}} \\
(\mathrm{au})\end{array}$ & $\begin{array}{l}\text { Exoplanet Mass } \\
\qquad M \sin i\left(\mathrm{M}_{\oplus}\right)\end{array}$ & $\begin{array}{c}\text { Semimajor Axis } \\
\text { (au) }\end{array}$ & Ref. \\
\hline GJ 1214 & 14.6 & M4.5 & 2935 & 53 & 0.096 & 6.4 & 0.0143 & $1,2,3$ \\
\hline GJ 876 & 4.7 & M5 & 3062 & 96.7 & 0.178 & $\begin{array}{l}615,194 \\
\mathbf{5 . 7}, 12.4\end{array}$ & $\begin{array}{c}0.208,0.130 \\
0.021,0.334\end{array}$ & $3,4,5$ \\
\hline GJ 581 & 6.3 & M5 & 3295 & 94.2 & 0.146 & $\begin{array}{c}15.9,5.4 \\
6.0,1.9\end{array}$ & $\begin{array}{c}0.041,0.073 \\
\mathbf{0 . 2 1 8}, 0.029\end{array}$ & $3,4,6$ \\
\hline GJ 436 & 10.3 & M3.5 & 3281 & 48 & 0.211 & 23 & 0.0287 & $3,4,7$ \\
\hline GJ 176 & 9.4 & M2.5 & 3416 & 38.9 & 0.262 & 8.3 & 0.066 & $3,4,8$ \\
\hline GJ $667 \mathrm{C}$ & 6.9 & M1.5 & 3327 & 105 & 0.172 & $5.7,4.4$ & $0.049, \mathbf{0 . 1 2 3}$ & $3,1,9$ \\
\hline GJ 832 & 4.9 & M1.5 & 3816 & $40^{\mathrm{b}}$ & 0.235 & $203,5.4$ & $3.6, \mathbf{0 . 1 6}$ & 3,4 \\
\hline HD 85512 & 11.2 & K6 & 4305 & 47.1 & 0.524 & 3.5 & 0.26 & $3,10,11$ \\
\hline HD 40307 & 12.9 & $\mathrm{~K} 2.5$ & 4783 & 48 & 0.674 & $\begin{array}{l}\mathbf{4 . 1}, \mathbf{6 . 7} \\
9.5, \mathbf{3 . 5} \\
\mathbf{5 . 1}, \mathbf{7 . 0}\end{array}$ & $\begin{array}{c}0.047,0.080 \\
0.132,0.189 \\
0.247,0.600\end{array}$ & $3,10,12$ \\
\hline$\epsilon$ Eri & 3.2 & $\mathrm{~K} 2$ & 5162 & 11.7 & 0.726 & $\sim 400$ & 3.4 & $3,10,13$ \\
\hline HD 97658 & 21.1 & $\mathrm{~K} 1$ & 5156 & 38.5 & 0.703 & 6.4 & 0.080 & $3,14,15$ \\
\hline
\end{tabular}

Notes.

"The habitable zone distance is defined as the average of the "runaway greenhouse" and "maximum greenhouse" limits (Kopparapu et al. 2014).

${ }^{\text {b }}$ GJ 832 has no published rotation period, we assume a relatively short period due to the persistence of UV flare activity in this star.

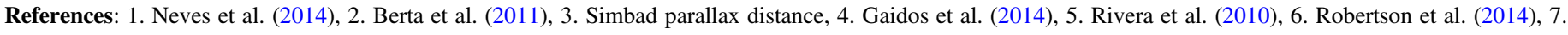

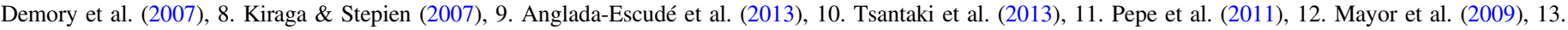
Donahue et al. (1996), 14. Valenti \& Fischer (2005), 15. Henry et al. (2011).

644 as a proxy for the GJ 581 stellar radiation field before the MUSCLES data were acquired. No weakly active $M$ dwarfs were bright enough to be observed by IUE outside of flares.

Two discrepancies between the spectra shown in Figure 2 are immediately apparent: (1) the "continuum flux" level of GJ 644 is approximately two orders of magnitude larger than GJ 581. The continuum level of the GJ 644 spectrum is approximately $2 \times 10^{-15} \mathrm{erg} \mathrm{cm}^{-2} \mathrm{~s}^{-1} \AA^{-1}$, which is consistent with the instrumental background equivalent flux for the IUE FUV low-resolution channel. The HST-COS background level is approximately $3 \times 10^{-17} \mathrm{erg} \mathrm{cm}^{-2} \mathrm{~s}^{-1} \AA^{-1}$, and no stellar FUV continuum is detected above this level in GJ 581. In Paper III, we show that approximately half of the M dwarfs in the MUSCLES sample have FUV continuum detections at this flux level. Therefore, the IUE spectrum includes a large amount of instrumental noise relative to the true flux upper limits in the FUV continuum. (2) The GJ 644 Ly $\alpha$ emission line is much brighter and broader than the reconstructed Ly $\alpha$ emission from GJ 581. The Ly $\alpha$ emission line in the IUE spectrum is almost entirely geocoronal airglow emission. The large $10^{\prime \prime} \times 20^{\prime \prime}$ oval IUE aperture admits 5000 times more geocoronal emission than STIS E140M observations using the 0 ". $2 \times 0$ "! 2 aperture, and the $I U E$ spectral resolution is too low to separate the interstellar absorption component from the stellar and geocoronal emission components. Therefore, IUE spectra cannot be used to compute the Ly $\alpha$ irradiance from faint, low-mass stars.

A comparison of the instrumental background and geocoronal airglow emission with the total 1160-1690 $\AA$ FUV flux from the IUE spectrum of GJ 644 indicates that $\approx 80 \%$ of the recorded counts come from non-stellar sources. This dramatic overestimate of the FUV flux in IUE M dwarf data would lead one to infer FUV/NUV flux ratios $\gtrsim 40$ when using the GJ 644 spectrum. This is much larger than the FUV/NUV flux ratios of $\sim 0.5-1$ that are found for $\mathrm{M}$ dwarf exoplanet host stars using the higher-sensitivity, higher-resolution HST data (Section 4.1). The MUSCLES database therefore provides researchers modeling atmospheric photochemistry and escape high-fidelity, HST-based, host star SEDs for their calculations.

\section{TARGETS AND OBSERVING PROGRAM}

\subsection{MUSCLES Target Stars}

The MUSCLES target list (Table 1) was chosen to cover a broad range of stellar types (K1V-M5V; $4 \mathrm{~K}$ dwarfs and $7 \mathrm{M}$ dwarfs), exoplanet masses, and semimajor axes, including most of the known $\mathrm{M}$ dwarf exoplanet host stars located within $d \lesssim 13$ pc (7/12), while excluding flare stars (e.g., GJ 674) that require intensive multi-wavelength monitoring to clear $H S T$ instrument safety protocols. The $\mathrm{M}$ dwarfs span a range of spectral types (from M1 to M5), a range of X-ray luminosity fractions $\left(\log _{10}\left(L_{X} / L_{\mathrm{Bol}}\right) \approx-5.1\right.$ to -4.4 , an indicator of activity level), and planetary systems ranging from Jupiters (GJ 832) to super-Neptunes (GJ 436) to super-Earths (GJ 1214). About $\sim 65 \%$ of the exoplanet host stars in our sample $(7 / 11)$ harbor super-Earths $\left(M_{\text {plan }}<10 M_{\oplus}\right.$; bold in Table 1$)$. In the brief summaries of the star-planetary systems given in the Appendix, we refer to the $M \sin i$ of the planets as their mass as a shorthand.

With the exception of $\epsilon$ Eri, the MUSCLES stars are not traditionally classified as active or flare stars (in contrast with widely studied M dwarf flare stars such as AU Mic or AD Leo). The stars in our sample are considered to be "optically inactive," based on their $\mathrm{H} \alpha$ absorption spectra (Gizis et al. 2002). However, the $\mathrm{Ca}$ II $\mathrm{H}$ and $\mathrm{K}$ emission line cores are a more straightforward means of diagnosing chromospheric activity in the optical (Gomes da Silva et al. 2011) because the $\mathrm{Ca}$ II emission line flux increases with activity while $\mathrm{H} \alpha$ first shows enhanced absorption before becoming a strong emission line with increasing activity. All of our stars with measured $\mathrm{Ca}$ II $\mathrm{H}$ and $\mathrm{K}$ profiles show weak but detectable emission (equivalent widths, $W_{\lambda}(\mathrm{Ca}$ II $)>0$; note that the references below present emission lines as positive equivalent widths, which is in opposition from the traditional convention), 


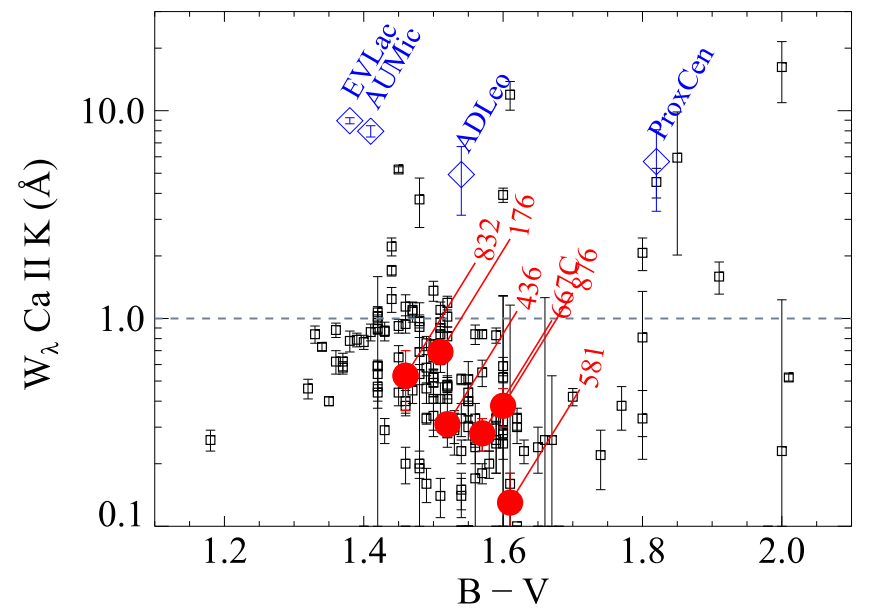

Figure 3. Ca II $\mathrm{K}$ equivalent width as a function of $(B-V)$ color (Rauscher \& Marcy 2006; Walkowicz \& Hawley 2009) for bright $M$ dwarfs and the MUSCLES M dwarf targets. The MUSCLES stars (red circles) are characterized as "weakly active" with Ca II activity indicators approximately an order of magnitude lower than traditional $M$ dwarf flare stars (blue diamonds, EV Lac, AU Mic, AD Leo, and Proxima Cen). Note that large, positive equivalent widths indicate stronger activity (emission line flux) in this presentation.

indicating that at least a low level of chromospheric activity is present in these stars (Rauscher \& Marcy 2006; Walkowicz \& Hawley 2009). In addition, Hawley et al. (2014) have shown that inactive $\mathrm{M}$ dwarfs display flares in their Kepler light curves, confirming that chromospheric activity is present on MUSCLES-type stars. Figure 3 shows the $\mathrm{Ca}$ II $\mathrm{K}$ line equivalent width as a function of $(B-V)$ color for six of the $M$ dwarfs in the sample, overplotted on the data from Rauscher \& Marcy (2006). The Ca II $\mathrm{K}$ emission is in the range $0.1<W_{\lambda}(\mathrm{Ca}$ II $)<0.8 \AA$, which is approximately an order of magnitude smaller than traditional flares stars EV Lac, AU Mic, AD Leo, and Proxima Cen (also shown in Figure 3). According to the M dwarf classification scheme of Walkowicz \& Hawley (2009), these stars have intermediate chromospheres and are referred to as "weakly active." In the Appendix, we present brief descriptions of each of the stars studied here. For more detailed descriptions of the stellar parameters of the MUSCLES target stars, we refer the reader to Loyd et al. (2016) and Youngblood et al. (2016).

\subsection{MUSCLES Observing Strategy}

Ultraviolet Observations, 100-3200 $\mathrm{A}$ - In order to obtain a full census of the UV emission incident on the HZs of lowmass stars, we require $H S T$ spectral coverage from 1150-3100 A: the G130M, G160M, and G230L modes of COS, and the G140M, E140M, E230M, E230H, and G230L modes of STIS provide spectral coverage across this bandpass. This combination of instrumental settings allows us to catalog the stellar emission lines that are relevant to the photoexcitation of the primary atmospheric constituents of the exoplanets in these systems. The $\mathrm{H}_{\text {I }} \mathrm{Ly} \alpha$ emission line dominates the total UV luminosity of M dwarfs (France et al. 2012a). We use the G140M mode of STIS with the $52^{\prime \prime} \times 0$ ". 1 slit to measure the Ly $\alpha$ profile for our $M$ dwarf targets. We have previously demonstrated that this technique can produce high-quality measurements of the local Ly $\alpha$ flux (France et al. 2013). For the brighter K dwarfs, we employ the STIS E140M mode, using the 0 !" $2 \times 0$ ". 06 slit to resolve the intrinsic line profile and minimize contamination by telluric $\mathrm{H}$ I. Resonant scattering of $\operatorname{Ly} \alpha$ in the local ISM requires that the line must be reconstructed to provide a reliable measure of the intrinsic Ly $\alpha$ radiation field in these exoplanetary systems. We direct the reader to Paper II for a detailed description of the Ly $\alpha$ reconstruction developed for the MUSCLES Treasury Survey (Youngblood et al. 2016).

In the FUV (except Ly $\alpha$ ), we use COS G130M (Si III $\lambda 1206$, $\mathrm{N}$ v $\lambda 1240, \mathrm{C}_{\text {III }} \lambda 1335$, and Si IV $\lambda 1400$ lines) and COS G160M ( $\mathrm{C}$ IV $\lambda 1550, \mathrm{He}$ II $\lambda 1640$, and $\mathrm{Al}$ II $\lambda 1671$ lines). The emission from $\mathrm{Ly} \alpha$, $\mathrm{Si}$ IV, $\mathrm{C}_{\mathrm{IV}}$, and $\mathrm{He}$ II is particularly interesting because these lines provide constraints on the Lyman continuum/EUV $(200 \lesssim \lambda \lesssim 900 \AA)$ irradiance in these systems (Linsky et al. 2014; Shkolnik et al. 2014). COS is essential for a moderate spectral resolution FUV line census as the lower effective area and higher detector background of STIS make observations of all but the very brightest emission line $\left(\mathrm{H}_{\mathrm{I}} \mathrm{Ly} \alpha\right)$ prohibitively time consuming. We use the medium-resolution ("M") COS modes to resolve chromospheric emission lines and maximize contrast from narrow spectral features.

At NUV wavelengths, we use STIS G230L $(\lambda>2200 \AA)$ to observe the NUV continuum, Fe II $\lambda 2400$ and $\lambda 2600$, and $\operatorname{Mg}$ II $\lambda 2800$, but take advantage of the superior sensitivity of the COS G230L mode to observe the 1750-2200 $\AA$ region that is important for the photodissociation of $\mathrm{O}_{2}$ and the production of $\mathrm{O}_{3}$. For targets that exceed the $\mathrm{G} 230 \mathrm{~L}$ bright-object limit (the K stars, $\epsilon$ Eri, HD 40307, HD 85512, and HD 97658), we use the higher-resolution E230M mode $(\lambda 1978+\lambda 2707$ settings) covering the $1800-3100 \AA$ bandpass. Target brightness limits dictated that we employ the STIS E230H mode for observations of the $\mathrm{Mg}$ II emission lines on the brightest $\mathrm{K}$ dwarf ( $\epsilon$ Eri).

The Ly $\alpha$ fluxes are combined with models of solar active regions (Fontenla et al. 2011) to estimate the EUV luminosity in the wavelength region $100-1170 \AA$ in $100 \AA$ bins (see Linsky et al. 2014 and Paper II). These EUV calculations were compared to the GJ 832 atmosphere model developed by Fontenla et al. (2016); we found that the 10-100 $\AA$ and 100-1000 $\AA$ bands differed by less than a factor of two. Given that no inactive $\mathrm{M}$ dwarf EUV observations exist as a reference point, we consider this intermodel agreement to be sufficient.

The MUSCLES observing plan was designed to study both the spectral and temporal variability of low-mass exoplanet host stars on the characteristic timescale for UV/optical variability (Kowalski et al. 2009). Using the most sensitive UV photon-counting mode on HST (COS G130M) and the time-tag capability of the COS microchannel plate detector (Green et al. 2012), we measure the $10^{4-5} \mathrm{~K}$ chromospheric and transition region activity indicators (using the $\mathrm{C}$ II, Si III, Si IV, and $\mathrm{Nv}$ emission lines) in $8 \mathrm{hr}$ intervals (5 contiguous spacecraft orbits for each star). Quasi-simultaneous X-ray observations were coordinated with these visits when possible (see below). While this strategy does not provide continuous coverage due to Earth occultation, it is optimized for constraining the types and frequency of flare behavior on low-mass exoplanet host stars. Since the characteristic timescale for UV/optical flare activity on M dwarfs is thought to be minutes to hours (Welsh et al. 2007; Kowalski et al. 2009; Loyd \& France 2014), our observations are ideal for quantifying the importance of flare activity to the local UV radiation field in these systems. 
With the above considerations in mind, we arranged the UV - visible observations for each target into campaigns comprising three HST visits: (1) COS G130M, (2) COS G160M + G230L, and (3) STIS G/E140M+G230L+G430L, all executed within a day of each other to mitigate uncertainties introduced by month or year timescale variations in the stellar flux. Between 9 and 13 total $H S T$ exposures were acquired for each star depending on the target brightness and the observing modes used. For a graphical description of which modes contribute at which wavelengths for each target, we refer the reader to Loyd et al. (2016).

$X$-ray Observations, $\quad 5-50 \AA \quad(2.5-0.25 \mathrm{keV})-\mathrm{We}$ used quasi-simultaneous Chandra and XMM-Newton observations of the MUSCLES stars to provide temporally consistent SEDs and to explore the wavelength-dependent behavior of stellar flares. The X-ray observations were coordinated with the COS G130M 5-orbit monitoring program (see above) to establish the longest possible simultaneous baseline over which to explore the panchromatic properties of $\mathrm{M}$ and $\mathrm{K}$ dwarf flares. We observed GJ 667C, GJ 436, GJ 176, and GJ 876 using the Chandra ACIS-S back-illuminated S3 chip. Owing to an HST safing event in 2015 June, the GJ 876 campaign was not simultaneous. We observed $\epsilon$ Eri and GJ 832 with XMMNewton because they are optically bright. $\epsilon$ Eri is by far the brightest X-ray source in our sample and provided a highquality RGS grating spectrum. $\epsilon$ Eri required use of the pn/ MOS Thick filters, while GJ 832 used the Medium filters. We also obtained photometry with the UVM2 filter for GJ 832. HD 85512 and HD 40307 were observed as part of a complementary XMM-Newton program (PI-A. Brown) using the same configuration as for GJ 832. For the stars without contemporaneous X-ray data, we obtained spectra from the Chandra and XMM-Newton archives (A. Brown et al. 2016, in preparation; Loyd et al. 2016). The coronal model fit to the X-ray data is

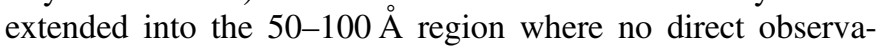
tions are available.

Optical Observations, 3200-6000 A-We carried out complementary ground-based observations as close in time as possible to the MUSCLES UV/X-ray observations. Our primary optical spectra came from the ARCES and DIS instruments on the Astrophysical Research Consortium (ARC) $3.5 \mathrm{~m}$ telescope at Apache Point Observatory (3700-10000 ̊, depending on the mode), the $2.15 \mathrm{~m}$ Jorge Sahade telescope at the Complejo Astronomico El Leoncito (Cincunegui \& Mauas 2004), and the FLOYDS intrument on the Las Cumbras Observatory Global Telescope Network (Brown et al. 2013). Multi-band optical photometry was acquired with the Apache Point Observatory $0.5 \mathrm{~m}$ ARCSAT telescope (with the Flarecam instrument) and the LCOGT. The optical data and their correlation with the spectral and temporal behavior of the high-energy emission will be presented in a future work by the MUSCLES team (A. Youngblood et al. 2016, in preparation). In order to calibrate the UV data with respect to visible/IR photospheric models and ground-based spectra, we also acquired short optical observations with STIS G430L or G430M (depending on target brightness) during each HST campaign.

Infrared Extension, $>6000 \AA$-Stellar atmosphere models considering only the photosphere (e.g., the PHOENIX models) are able to reproduce the peak and Rayleigh-Jeans tail of the stellar SED given the correct prescription for the effective temperature and abundances of atoms and molecules (Allard \&
Hauschildt 1995; Husser et al. 2013). We employ PHOENIX models, matched to the Tycho $B$ - and $V$-band fluxes, to calibrate the optical spectroscopy from HST-STIS and fill out the panchromatic SEDs from the red-optical to the mid-IR ( 0.6-5.5 $\mu \mathrm{m}$; Loyd et al. 2016). The red-optical and infrared fluxes are important as they make up the majority of the bolometric luminosity from cool stars, regulate the effective surface temperature of orbiting planets, and provide the reference for the activity indicators (e.g., $L(\mathrm{~N} \mathrm{v}) / L_{\mathrm{Bol}}$ ) presented in this work.

\section{RESULTS: $5 \AA$ TO $5 \mu \mathrm{m}$ IRRADIANCE SPECTRA}

The primary goal of the MUSCLES Treasury Survey is to develop a uniform set of irradiance spectra to support the study of extrasolar planets orbiting low-mass stars. The X-ray through optical observations are described above, a description of the analysis required to complete "missing" parts of the SED (intrinsic Ly $\alpha$ line profiles and EUV fluxes) is presented in Paper II (Youngblood et al. 2016), and a detailed discussion of the creation of the panchromatic radiation fields is given in Paper III of this series (Loyd et al. 2016). In the following subsections, we present both quantitative and qualitative descriptions of the SEDs, including the behavior of the broadband fluxes as a function of stellar effective temperature and HZ location, the distribution of stellar emission lines observed in the HST spectra, the use of individual spectral tracers to estimate the broadband energetic radiation fluxes, and an initial description of the temporal variability of the MUSCLES Treasury data set.

For the results presented below, we used both broadband and emission line fluxes. The broadband fluxes are simply integrals of the panchromatic SED over the wavelength region of interest. Ly $\alpha$ fluxes are the wavelength-integrated reconstructed line profiles obtained by Youngblood et al. (2016). We measured the metal emission lines by employing a Gaussian line-fitting code that takes into account the linespread function of the instrument used to create that portion of the spectrum. For instance, the $\mathrm{N}$ vand $\mathrm{C}$ ivlines are fit with a single-component Gaussian emission line convolved with the appropriate wavelength-dependent $H S T$-COS line-spread function $^{21}$ (France et al. 2012b). Some emission lines are clearly better described by a two-component model (Wood et al. 1997), but this only applies to a small number of emission lines in the sample and we assume a single Gaussian for the results presented in this paper. $\mathrm{Mg}$ II emission line fluxes assume an unaltered Gaussian line-shape for both COS and STIS observations. As most of the $\mathrm{Mg}$ II observations were made with the low-resolution G230L modes of COS and STIS, interstellar absorption correction is not possible for all of the targets, and so the $\mathrm{Mg}$ II line fluxes are presented as measured. Figures 4 and 5 show samples FUV (1328-1410 $)$ ) and NUV (2789-2811 $\AA$ ) spectral regions at full resolution with the prominent emission lines labeled.

\subsection{Broadband Fluxes}

Figure 6 shows a montage of the full $5 \AA-5 \mu \mathrm{m}$ SEDs for the 11 MUSCLES exoplanet host stars, binned to $5 \AA$ pixel $^{-1}$ for

\footnotetext{
${ }^{21}$ The COS line-spread function experiences a wavelength-dependent nonGaussianity due to mid-frequency wave-front errors produced by the polishing errors on the $H S T$ primary and secondary mirrors; http://www.stsci.edu/hst/ $\cos /$ documents/isrs/.
} 


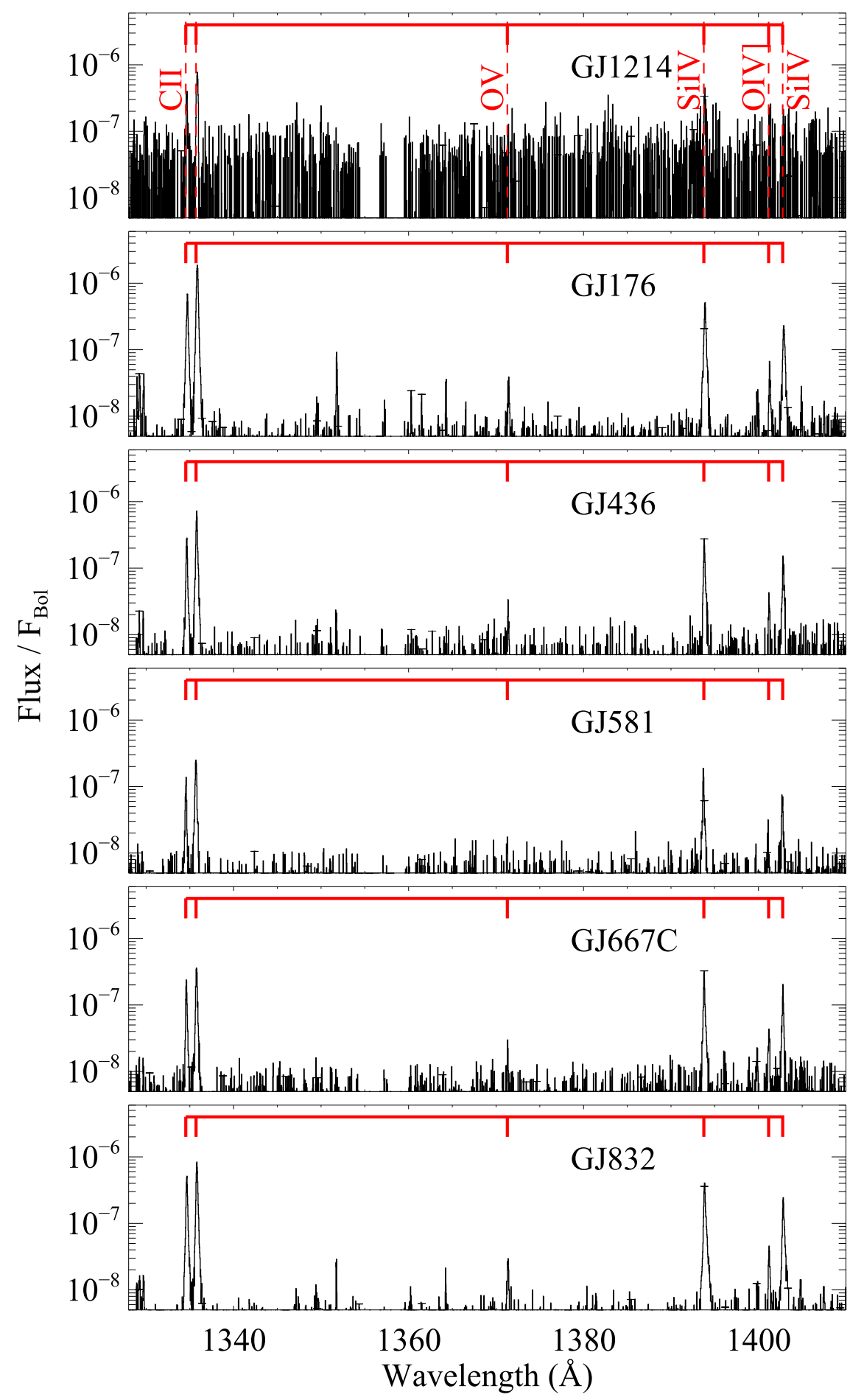

Figure 4. (a) A sample of the FUV spectra (1328-1410 ̊) of each MUSCLES target. The flux spectra are normalized by their total bolometric luminosity. These spectra are the coadded observations acquired with the HST-COS G130M grating over five consecutive orbits, designed to produce both high S/N FUV spectra and to characterize the energetic flare frequency on typical low-mass exoplanet host stars (Section 4.4). Emission lines formed at temperatures from roughly $10^{4}-10^{5} \mathrm{~K}$ are labeled. The broad features near 1356 and $1358 \AA$ are geocoronal O I]. (b) Same as Figure 4(a).

display and divided by the bolometric luminosity. The bolometric luminosity used in this work is simply the integral of the complete SED for each star (including a calculated Rayleigh-Jeans tail at $\lambda>5.5 \mu \mathrm{m}$; Loyd et al. 2016). Many of the component spectra have spectral resolutions much higher than this (resolving powers of $R>15,000$ for all of the FUV data and up to $R=114,000$ for STIS E230H observations of $\epsilon$ Eri), and both full-resolution and binned data sets are available on the MAST HLSP archive. ${ }^{22}$ As discussed in the Introduction, the broadband behavior of the stellar SED can provide general insights into the relevant heating and photochemical rates for all types of exoplanet atmospheres.

We define the FUV flux, $F(\mathrm{FUV})$, as the total stellar flux integrated over the 912-1700 $\AA$ bandpass, including the

\footnotetext{
$\overline{22}$ https://archive.stsci.edu/prepds/muscles/
} 


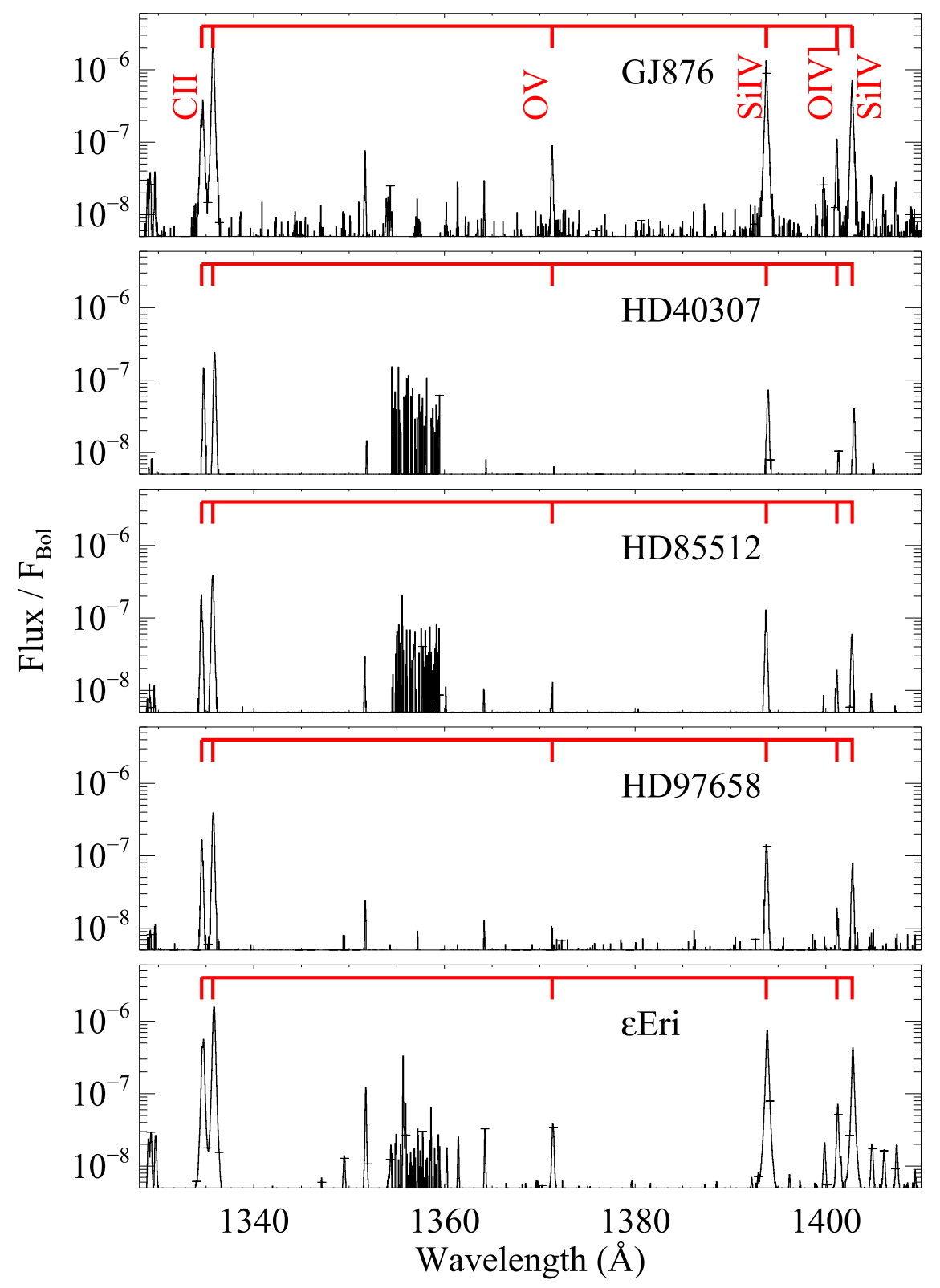

Figure 4. (Continued.)

reconstructed $\mathrm{Ly} \alpha$ emission line, $\mathrm{FUV}=(F(\mathrm{Ly} \alpha)+F$ $(1170-1210+1220-1700)+F(912-1170)) . F(\mathrm{Ly} \alpha)$ is the reconstructed Ly $\alpha$ line flux, $F(1170-1210+1220-1700)$ is the non-Ly $\alpha$ flux directly measured by HST-COS and STIS, and $F$ (912-1170) is the "Lyman Ultraviolet" emission constructed from the solar active region relations for low-mass stars presented by Linsky et al. (2014). On average, Ly $\alpha$ contributes $83 \%( \pm 5 \%)$ to the total FUV flux. $F(\mathrm{NUV})$ are the combined HST spectra integrated over 1700-3200 А. The fractional luminosities in each band as well as key emission lines are given in Table 3 .

Figure 7 shows the FUV/NUV flux ratio as a function of the stellar effective temperature. The FUV/NUV flux ratio is in the range $0.5-0.7$ for the latest $M$ stars (GJ 1214 and GJ 876), is in the range $0.2-0.4$ for M1 to M3 stars, declines to 0.04 by mid$\mathrm{K}$, and is $\lesssim 0.01$ for spectral types $\mathrm{K} 2$ and earlier. France et al. (2013) have previously shown that the FUV/NUV ratio drops to $\sim 10^{-3}$ for solar-type stars. The trend in FUV/NUV with effective temperature is largely driven by the large increase in photospheric emission moving into the NUV spectral bandpass with increasing effective temperature. Large FUV fluxes dissociate $\mathrm{O}_{2}$ to generate $\mathrm{O}_{3}$. The photospheric NUV photons destroy photochemically produced ozone and keep the atmospheric $\mathrm{O}_{3}$ mixing ratios low on Earth-like planets around solar-type stars in the absence of a disequilibrium process such as life.

The XUV flux (5-911 $\AA$ ), the combination of the observed and modeled soft X-ray flux (usually $2.5-0.125 \mathrm{keV}$, or $\approx 5-100 \AA)^{23}$ and the calculated EUV flux (100-911 $\mathrm{A}$ ), is an important heating agent on all types of planets. XUV irradiance

\footnotetext{
${ }^{23}$ We do not include hard X-rays in our panchromatic SEDs as the observations are not easily obtainable and the photoionization cross-sections for most of the relevant atmospheric constituents decline at energies higher than EUV + soft-X-rays.
} 


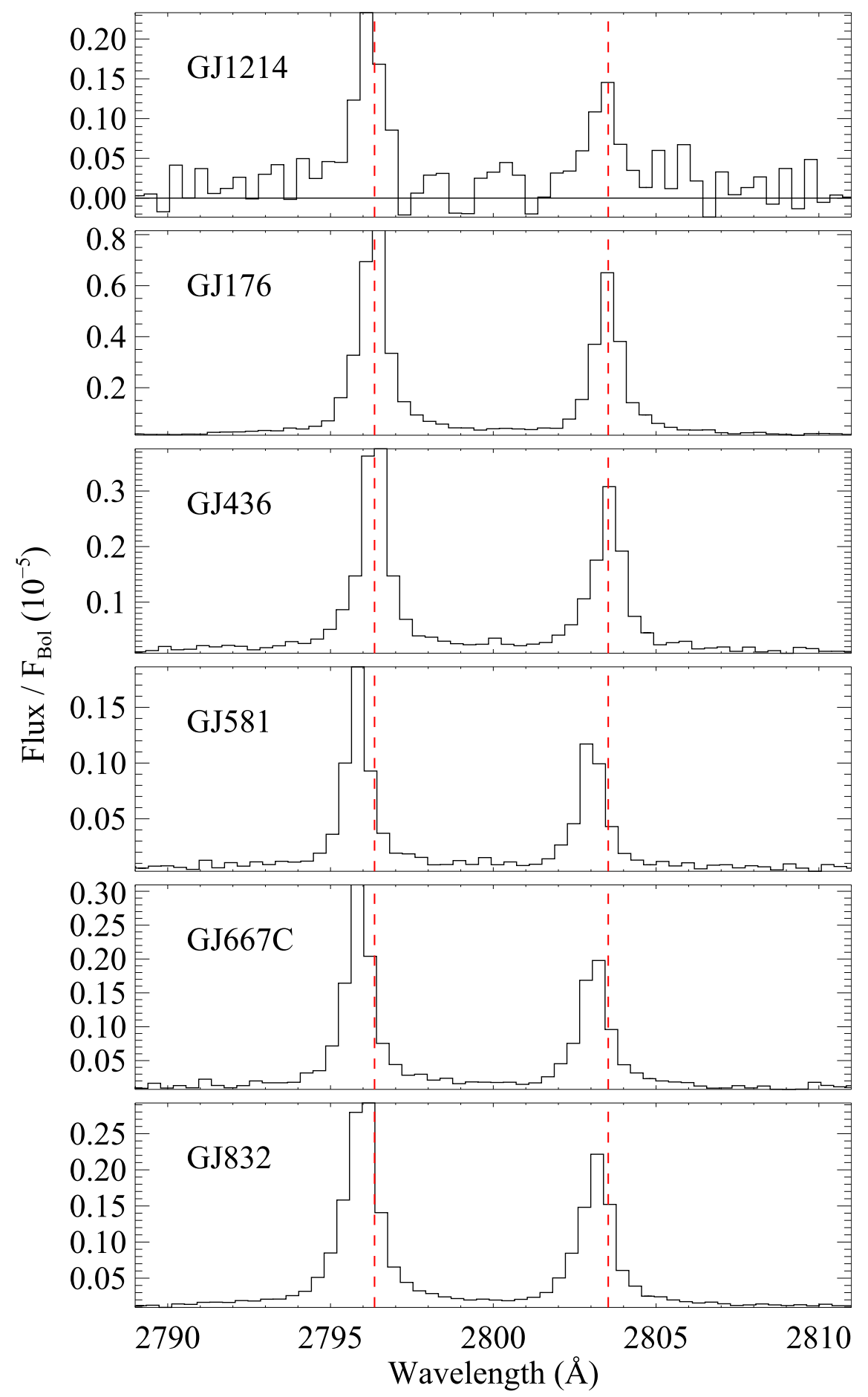

Figure 5. (a) NUV spectra (2789-2811 $⿱$ ) of each of the MUSCLES targets, centered on the Mg II doublet. The spectra are normalized by their total bolometric luminosity. These spectra were taken primarily with the COS G230L grating observations in order to show a uniform comparison. For the brighter $\mathrm{K}$ stars, we display higher-resolution HST-STIS E230M and E230H observations. At higher resolution, one can resolve the absorption from interstellar $\mathrm{Mg}^{+}$ions. Dashed lines identify the $\mathrm{Mg}$ II rest wavelengths. (b) Same as Figure 5(a).

is particularly important for short-period planets (Lammer et al. 2009). Figure 8 shows the relative XUV and FUV luminosities as a function of effective temperature for the MUSCLES stars. The total XUV and FUV luminosities are shown to be well-correlated with stellar effective temperature (and therefore stellar mass) and the fractional XUV and FUV luminosities are in the range $10^{-5} \lesssim L$ (band) $/ L_{\text {bol }} \lesssim 10^{-4}$ with no dependence on stellar effective temperature. For comparison, the disk-integrated quiet Sun (Woods et al. 2009) has $L(\mathrm{XUV}) / L_{\mathrm{Bol}}=2 \times 10^{-6}$ and $L(\mathrm{FUV}) / L_{\mathrm{Bol}}=1 \times 10^{-5}$, respectively. Note, however, that the solar $L(\mathrm{FUV})$ contains a contribution from the photosphere, whereas the photospheric contribution is negligible for $\mathrm{M}$ and $\mathrm{K}$ dwarfs. From the perspective of integrated planetary atmosphere mass-loss over time, our measurements represent only a conservative lowerlimit as the stellar XUV radiation was likely factors of 10-100 

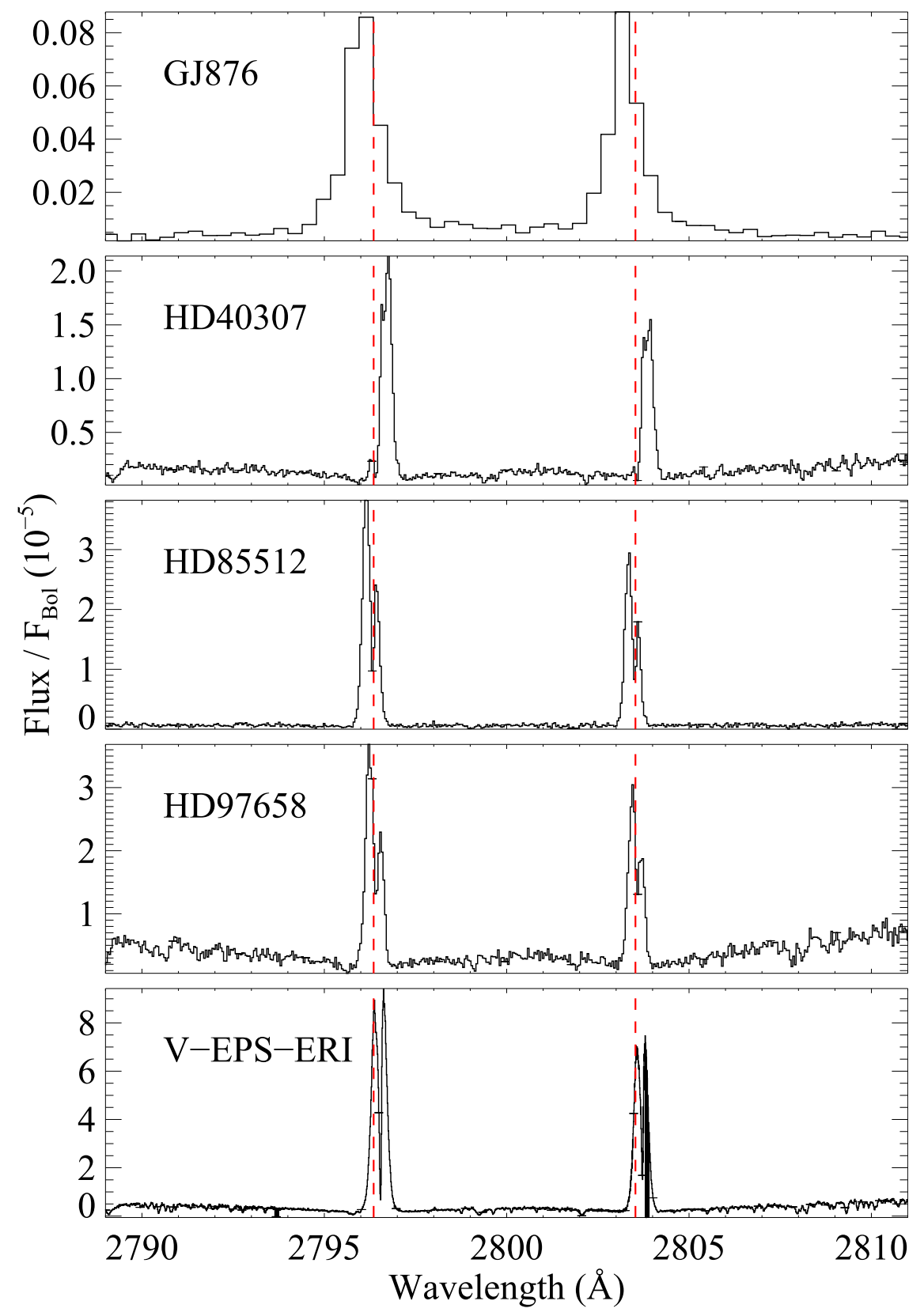

Figure 5. (Continued.)

times higher during the star's younger, more active periods. The active periods of $M$ stars are more prolonged than the equivalent "youthful magnetic exuberance" (Ayres \& France 2010) period of solar-type stars (e.g., West et al. 2008). Additionally, it is likely that the stellar mass-loss rates are higher earlier in their evolution (Wood et al. 2005a), increasing the potential planetary mass-loss.

We can combine these results into a comprehensive picture of the energetic radiation environment in the HZs around the low-mass stars. Figure 9 shows the FUV/NUV ratios, the total FUV fluxes, and the total XUV fluxes at the HZ orbital distances for each of our targets. The plots show the average $\mathrm{HZ}$ distance for each star, $\left\langle r_{\mathrm{HZ}}\right\rangle$, computed as the mean of the "runaway greenhouse" and "maximum greenhouse" limits to the HZ presented by Kopparapu et al. (2014). The error bars on $\left\langle r_{\mathrm{HZ}}\right\rangle$ represent these extrema. One observes the two orders of magnitude decline of the FUV/NUV ratio from 0.1 to 0.7 au, mainly driven by the stellar effective temperature dependence described above. The FUV HZ fluxes show a weak trend of increasing flux with increasing $r_{\mathrm{HZ}}$, however, both the $\mathrm{M}$ dwarf and $\mathrm{K}$ dwarf samples have a factor of roughly 5 dispersion at a given $\mathrm{HZ}$ distance. For example, while it is possible for the absolute FUV flux to be a factor of 10 greater at 0.7 au than at $0.15 \mathrm{au}$, it is also possible that the FUV flux at $0.15 \mathrm{au}$ is greater than at 0.7 au. A similar dispersion is seen in the XUV fluxes, and there is no statistically significant change in the XUV flux across the HZ (Table 2). Therefore, the average FUV and XUV fluxes in the HZs of $\mathrm{M}$ and $\mathrm{K}$ dwarfs are $10-70 \mathrm{erg} \mathrm{cm}^{-2} \mathrm{~s}^{-1}$. We emphasize that the activity level of the individual star must be considered, and direct observations are preferable when available. In Section 4.3, we will discuss the correlation of the broadband fluxes with specific emission line measurements to 

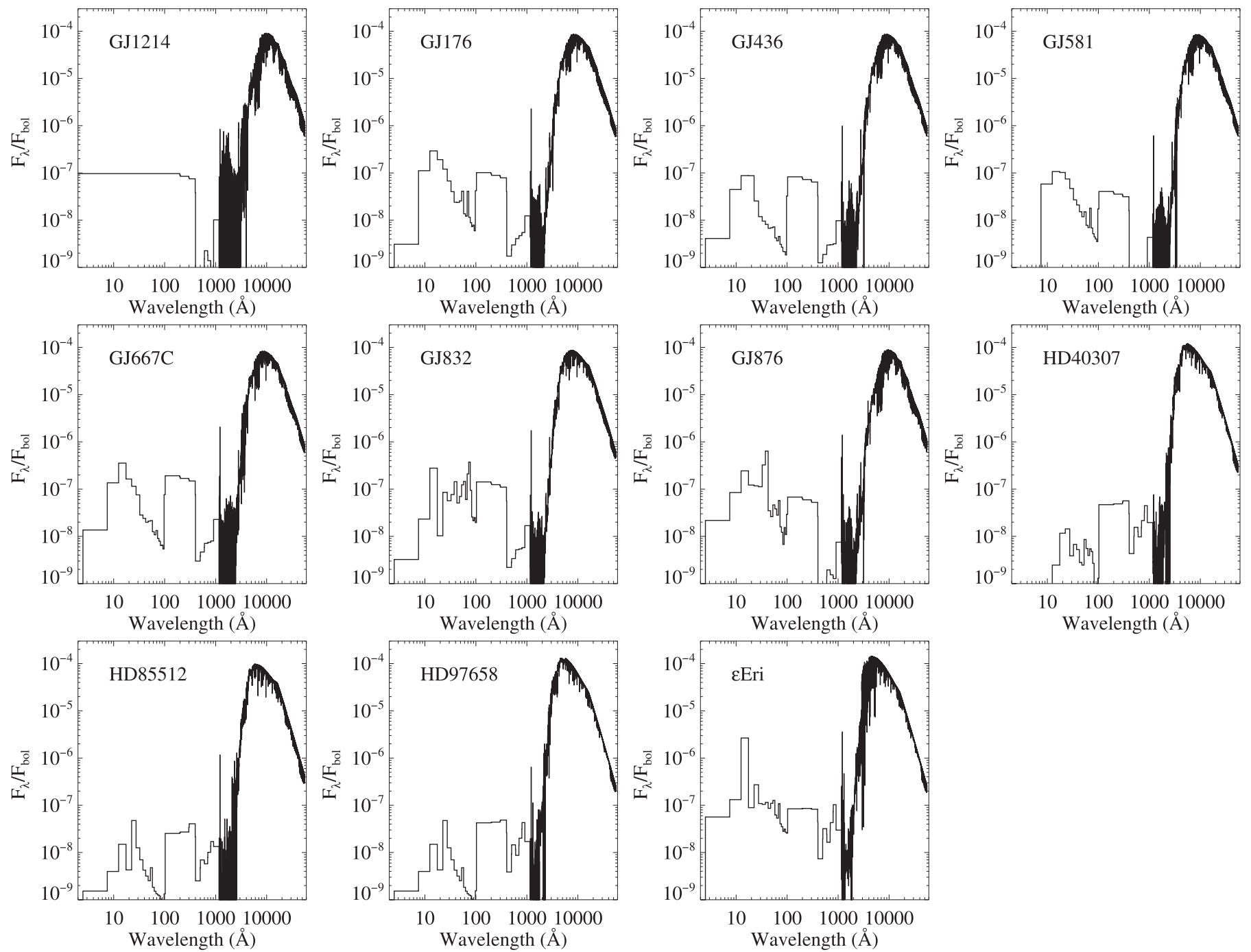

Figure 6. Panchromatic SEDs of the MUSCLES targets, binned to $5 \AA$ A per pixel for display purposes. H I Ly $\alpha$ ( $\lambda 1216 \AA$ ) is observed above the surrounding UV emission and contiuum features. Both native and 1 A resolution SEDs are available on the MAST High-level Science Products site: https://archive.stsci.edu/prepds/ muscles/.

simplify the characterization of the broadband fluxes from single emission line flux measurements.

\subsection{0-3200 A Stellar Emission Lines: The Ubiquity of UV Emission}

Chromospheric and transition region emission lines are observed in all of the MUSCLES spectra, which argues that all exoplanet host stars with spectral type M6 and earlier have UVactive atmospheres (Figures 4 and 5). This seems to rule out photosphere-only models of cool stars and indicates that the chromospheric, transition region, and coronal emission must be included for accurate modeling of the atmospheres of planets orbiting these stars. Bright emission lines in the MUSCLES spectra with chromospheric formation temperatures in the range $\sim(4-30) \times 10^{3} \mathrm{~K}$ include $\mathrm{H}_{\text {I }} \mathrm{Ly} \alpha, \mathrm{Si}_{\text {III }} \lambda 1264,1526$, 1808, C ІІ $\lambda \lambda 1334,1335, \mathrm{Al}$ ІІ $\lambda 1671, \mathrm{Fe}$ II multiplets near 2400 and $2600 \AA$, and $\mathrm{Mg}$ II $\lambda \lambda 2796,2803$ (see, e.g., the M dwarf contribution functions presented by Fontenla et al. 2016). We observe many transition region lines with formation temperatures in the range $\sim(40-200) \times 10^{3} \mathrm{~K}$, including the $\mathrm{C}_{\text {III }} 1175$ multiplet, Si III $\lambda 1206 ， \mathrm{O} v \lambda 1218 ， 1371 ， \mathrm{~N} v \lambda \lambda 1239 ， 1243$,
O Iv] $\lambda 1401 ， \operatorname{Si}$ Iv $\lambda \lambda 1394 ， 1403 ， \mathrm{C}$ Iv $\lambda \lambda 1548 ， 1550$, and He II $\lambda 1640$. The coronal iron lines, Fe XII $\lambda 1242$ and Fe xxi $\lambda 1354$ (Ayres et al. 2003), are observed in a fraction of HST-COS observations (7/11 stars show Fe XII emission lines while only GJ 832 and GJ 876 show Fe XXI emission lines). Taken together with the X-ray observations, these highly ionized iron lines demonstrate the presence of coronal gas in the atmospheres of all of our stars and enable an alternative calculation of the EUV irradiance using emission measure techniques (Sanz-Forcada et al. 2011; Chadney et al. 2015). We will present a detailed discussion of the atmospheric kinematics derived from emission line parameters in an upcoming work (J. Linsky et al. 2016, in preparation).

A major complication in previous attempts to assemble panchromatic radiation fields, particularly those of $\mathrm{M}$ dwarfs, is the flux variability between observations separated by years (e.g., comparing IUE spectra from the 1980s with ROSAT X-ray data from the 1990s and HST observations of Ly $\alpha$ from the 2000s; Linsky et al. 2013, 2014). One of the goals of the MUSCLES Treasury Survey is to obtain multi-wavelength observations close in time to minimize this large systematic uncertainty. In the MUSCLES observing strategy, emission 


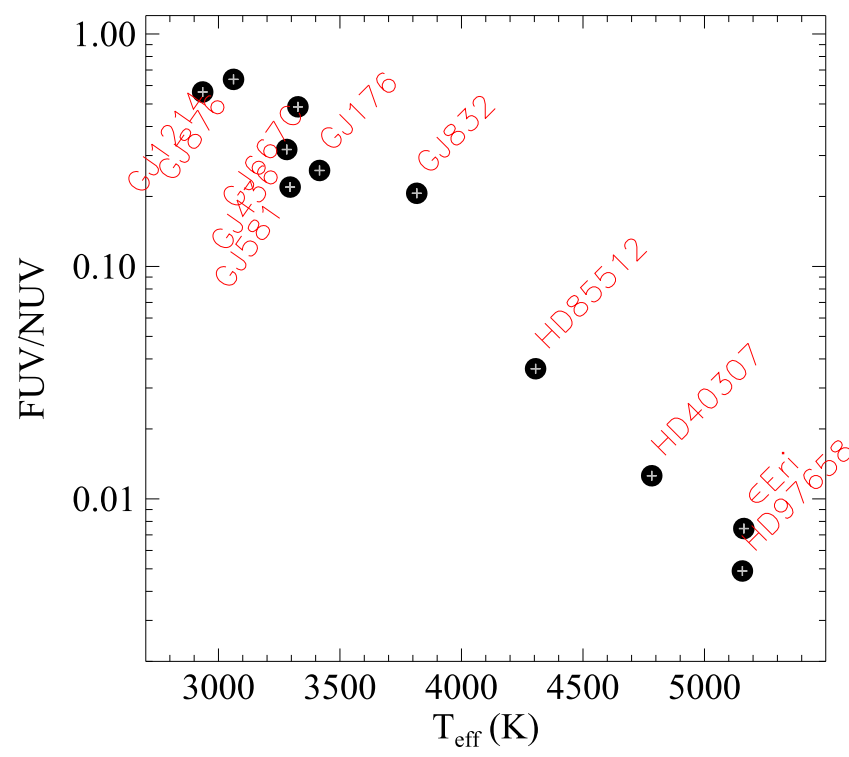

Figure 7. FUV/NUV $(912-1700 \AA) /(1700-3200 \AA)$ stellar flux ratio decreases with $T_{\text {eff }}$ by a factor of $\sim 100$ from mid-M dwarfs to early $\mathrm{K}$ dwarfs. This relationship is largely driven by the increasing photospheric flux in the NUV band of hotter stars. The FUV/NUV flux ratio has been shown to impact oxygen chemistry on Earth-like planets in the HZ (Hu et al. 2012; Tian et al. 2014).

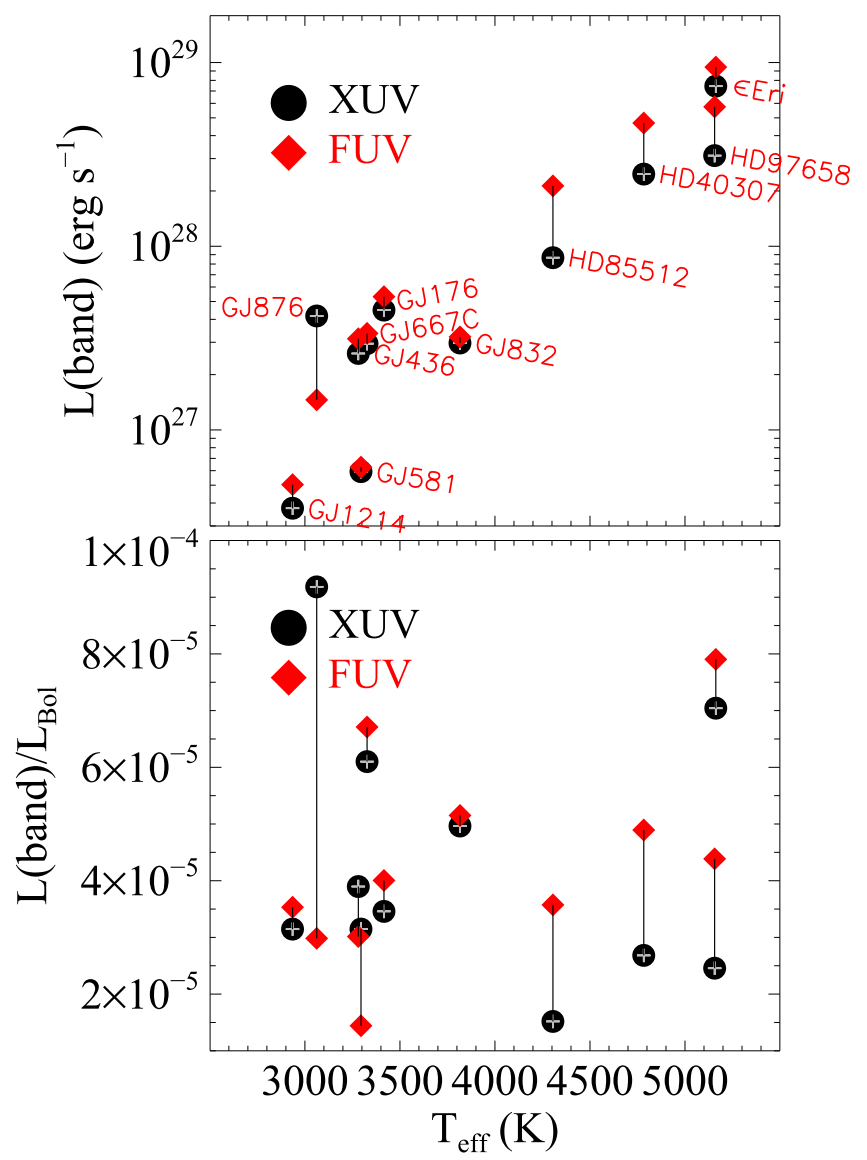

Figure 8. Total UV luminosity is proportional to the effective temperature (and therefore stellar mass), whereas the fractional luminosity is roughly constant across the sample. (Top) The total XUV (5-911 $⿱$ ) ) luminosity and total FUV (912-1700 A) luminosity increase with stellar effective temperature. (Bottom) The fractional luminosities in the XUV and FUV bands are in the range $10^{-5}$ $10^{-4}$ for all of the MUSCLES targets. The black circles represent the XUV luminosity while the red diamonds represent the FUV luminosity.

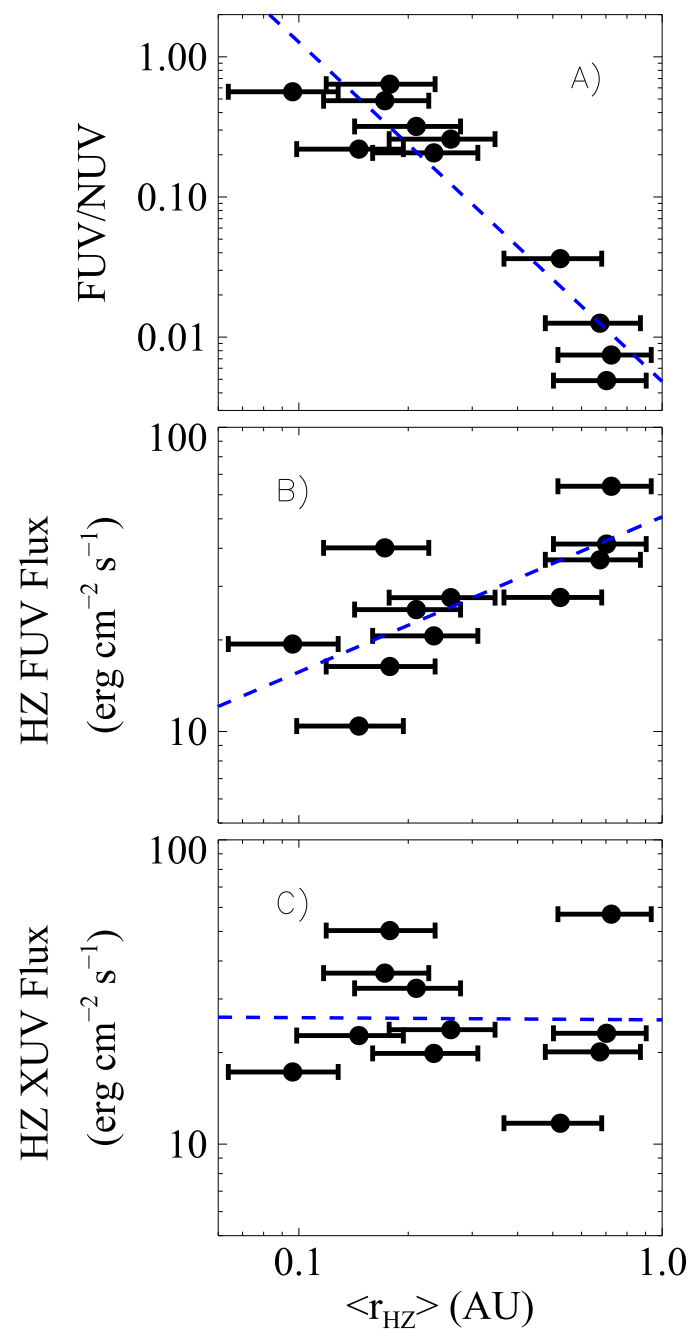

Figure 9. Evolution of the ultraviolet radiation environment over the habitable zone for low-mass stars. The habitable zone distance, $\left\langle r_{\mathrm{HZ}}\right\rangle$, is defined as the average of the "runaway greenhouse" and "maximum greenhouse" limits (Kopparapu et al. 2014), and the error bars show these extrema. The FUV/ NUV ratio increases by a factor of 100 from 0.7 to 0.1 au (panel A), while the average absolute FUV (panel B) flux decrease by factors of three to five toward the inner habitable zone. Note that the scatter in the FUV flux at any given HZ radius is at least as large as the correlations indicated by the log-log fits shown as the blue dashed lines. The average absolute EUV (panel C) flux is constant across the HZ.

Table 2

Emperical Log-Log Relations, $\log _{10} y=\left(m \times \log _{10} x\right)+b$

\begin{tabular}{lccr}
\hline \hline$y$ & $x$ & $m$ & \multicolumn{1}{c}{$b$} \\
\hline$L(\mathrm{FUV})^{\mathrm{a}}$ & $L(\mathrm{C}$ IV $)$ & $1.12 \pm 0.27$ & $-1.01 \pm 6.86$ \\
$L(\mathrm{FUV})$ & $L(\mathrm{Mg}$ II $)$ & $0.75 \pm 0.06$ & $7.56 \pm 1.52$ \\
$L(\mathrm{XUV})$ & $L(\mathrm{C}$ IV $)$ & $0.97 \pm 0.19$ & $2.72 \pm 4.91$ \\
$L(\mathrm{XUV})$ & $L(\mathrm{Mg}$ II $)$ & $0.58 \pm 0.08$ & $11.98 \pm 2.12$ \\
\hline FUV $/$ NUV & $\left\langle r_{\mathrm{HZ}}\right\rangle$ & $-2.42 \pm 0.30$ & $-2.31 \pm 0.18$ \\
HZ FUV Flux & $\left\langle r_{\mathrm{HZ}}\right\rangle$ & $0.51 \pm 0.16$ & $1.71 \pm 0.10$ \\
HZ XUV Flux $^{\mathrm{b}}$ & $\left\langle r_{\mathrm{HZ}}\right\rangle$ & $-0.01 \pm 0.22$ & $1.41 \pm 0.14$ \\
\hline$L(\mathrm{~N} \mathrm{v}) / L_{\mathrm{Bol}}$ & $M_{\text {plan }} / a_{\text {plan }}$ & $0.64 \pm 0.24$ & $-8.53 \pm 0.56$
\end{tabular}

Notes.

${ }^{\text {a }}$ All luminosities are in units of $\left(\mathrm{erg} \mathrm{s}^{-1}\right)$.

${ }^{\mathrm{b}}$ Habitable zone fluxes are in units of $\left(\mathrm{erg} \mathrm{cm}^{-2} \mathrm{~s}^{-1}\right),\left\langle r_{\mathrm{HZ}}\right\rangle$ and $a_{\text {plan }}$ in units of (au), $M_{\text {plan }}$ in units of $\left(M_{\oplus}\right)$. 


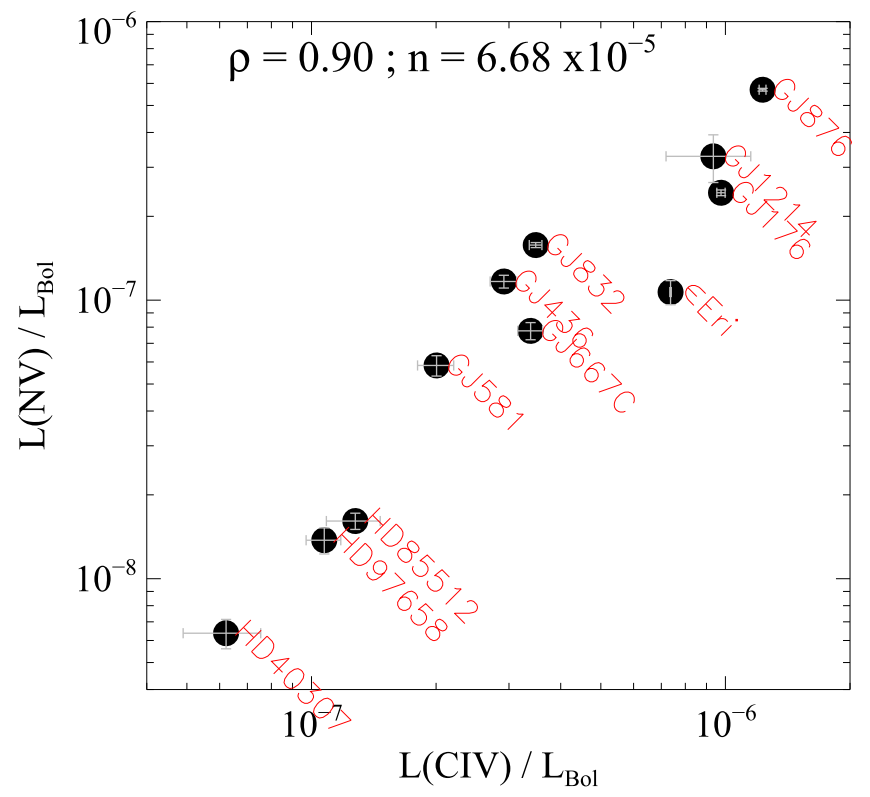

Figure 10. Comparison of the fractional $\mathrm{C}$ IV and $\mathrm{N} v$ luminosities (relative to the bolometric luminosity) of the MUSCLES samples. The Pearson correlation coefficient $(\rho)$ and a statistical measure of the possibility of no correlation $(n)$ are shown at the top of the panel, quantifying the obvious strong correlation between the two emission lines. This plot shows that the observation-averaged stellar fluxes from similar formation temperature ranges do not vary significantly on the $\sim 1$ day timescales separating the MUSCLES observations.

lines with similar formation temperatures were acquired on different $H S T$ visits (due to their inclusion on different $H S T$ grating modes) separated by $18-48 \mathrm{hr}$. The overlap in formation temperature and spectral coverage between adjacent modes facilitates scaling over calibration variations (Loyd et al. 2016) and smaller day-to-day variations.

This approach has been successful-Figure 10 shows the relationship of the fractional luminosity in the transition region ions $\mathrm{Nv}$ and $\mathrm{C}$ Iv. These lines have formation temperatures within a factor of two of each other (between $(1-2) \times 10^{5} \mathrm{~K}$ ) and have been shown to be tightly correlated in numerous astrophysical plasmas, including the atmospheres and accretion columns around young stars (Oranje 1986; Ardila et al. 2013; France et al. 2014). The $\mathrm{N} v$ versus $\mathrm{C}$ Iv correlation is very well maintained over the MUSCLES sample despite the nonsimultaneous observations, with a Pearson correlation coefficient of 0.90 ( $\rho$ in the legend of Figure 10) and a probability of no correlation of $6.0 \times 10^{-5}$ ( $n$ in the legend of Figure 10). Because we do not exclude discrete impulsive flares, the MUSCLES spectra can be considered to be an accurate snapshot of the average stellar spectrum (averaged on the timescale of hours-todays). Variability on the timescales of the solar cycle (or stellar cycles, years) will be addressed by developing XUV-optical tracer correlations and carrying out long-term monitoring from ground-based facilities (Section 1.3).

The emission lines in the MUSCLES stars also show an evolution of decreasing fractional luminosity of the transition region with increasing effective temperature. Figure 11 (top) shows the fractional $\mathrm{Nv}$ luminosity as a function of the effective temperature. Given that the total XUV and FUV factional luminosities are approximately constant with effective temperature (Figure 8, lower), this suggests that the upper
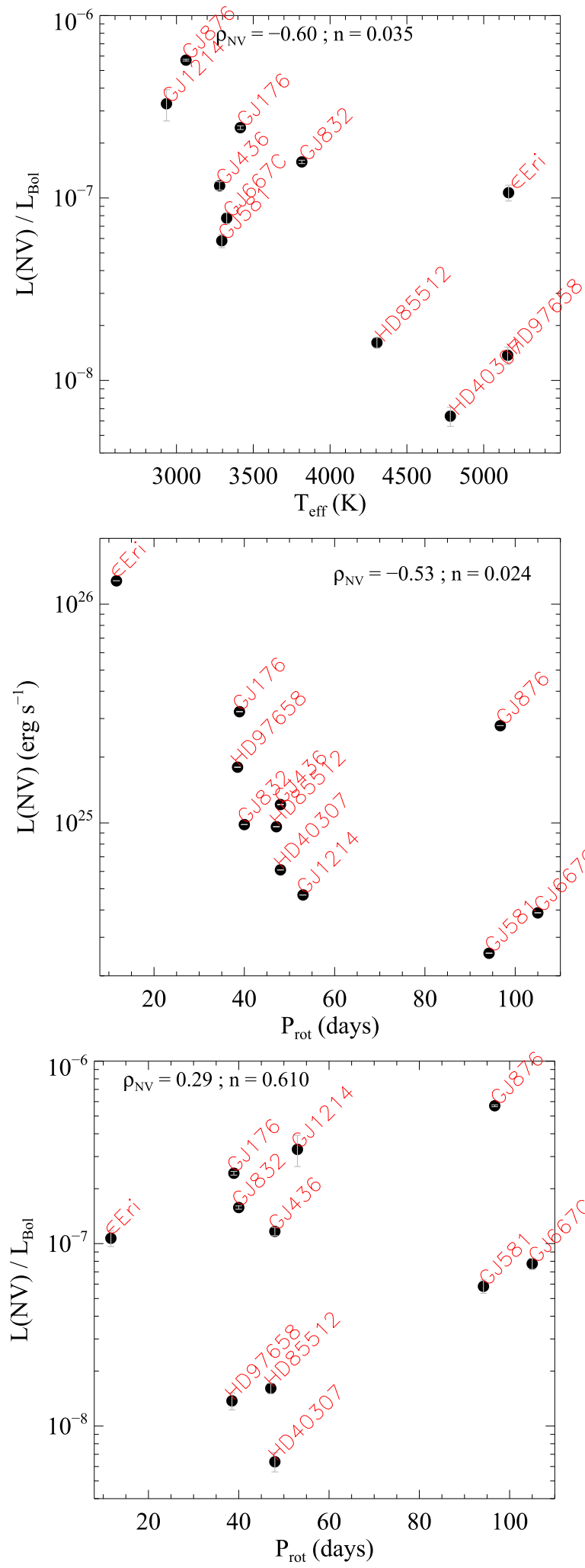

Figure 11. (Top) The transition region activity, traced here by the fractional $\mathrm{N} v$ luminosity (relative to the bolometric luminosity), displays a weak anticorrelation with stellar effective temperature. Note that $\epsilon$ Eri is the only "active" star in the MUSCLES sample. The middle plot shows that the total $\mathrm{N} \mathrm{v}$ luminosity may decline with rotation period, with the exception of GJ 876 (which is influenced by strong flares). (Bottom) There is no correlation between the fractional hot gas luminosity and the stellar rotation period. 
transition region activity (traced by $\mathrm{N} v$ ) declines faster than the rest of the upper atmosphere (e.g., the chromosphere and corona), which may be related to the pressure-density structure of the stellar atmosphere changing with mass (Fontenla et al. 2016). While the Pearson correlation coefficient is only $-0.60\left(n=3.5 \times 10^{-2}\right)$, this is skewed by the inclusion of $\epsilon$ Eri, the only active star in the sample. Excluding $\epsilon$ Eri, the coefficient becomes $-0.65\left(n=9.1 \times 10^{-3}\right)$. The middle plot in Figure 11 shows the total $\mathrm{Nv}$ luminosity with the stellar rotation rate, suggesting a period-activity relation analogous to the well-studied relationship in solar-type stars (Ribas et al. 2005; see also Engle \& Guinan 2011 for M dwarf X-ray evolution). The notable exception is GJ 876, with an $\mathrm{Nv}$ luminosity approximately an order of magnitude larger than expected based on its rotational period. This is partially the result of the strong flare activity on this star (see Section 4.4), and also suggests that the period is not well determined for this star. Excluding GJ 876, the Pearson and Spearman coefficients are -0.62 and -0.92 , respectively. If GJ 876 fell on this trend, then we would expect a rotation period closer to $\sim 40$ days. Interestingly, there is no trend in the fractional $\mathrm{N} \mathrm{v}$ luminosity with stellar rotation rate (Figure 11, bottom).

\subsection{The Correlation between $C$ IV and $M g$ II Fluxes and Broadband Luminosities}

Another goal of the MUSCLES Treasury Survey is to identify individual spectral tracers that can serve as proxies for the broadband fluxes from low-mass stars so that large, resource-intensive projects may not be required to obtain accurate estimates for the energetic radiation environments around exoplanets in the future. This anticipates a time beyond the current suite of UV and X-ray observatories (e.g., XMM, Chandra, and HST) capable of making these measurements, and a time when the sheer number of potentially habitable planets around low-mass stars precludes a detailed panchromatic characterization of every target (possibly after the TESS mission). In this case, simple relationships for acquiring reasonably accurate broadband stellar flux measurements will be essential for accurately modeling the atmospheric spectra from these worlds when they are acquired by future flagship missions in the 2020s, 2030s, and 2040s. Below, we describe the relationship between the XUV and FUV luminosities of the MUSCLES stars with the most prominent FUV and NUV emission lines, $\mathrm{Mg}$ II and $\mathrm{C}_{\text {IV. }}{ }^{24}$ In a future work, we will explore ground-based tracers of the UV radiation environment (e.g., Ca II $\mathrm{H} \& \mathrm{~K}$ fluxes and equivalent widths from our contemporaneous ground-based observations; A. Youngblood et al. 2016, in preparation).

Figure 12 shows the relationship between the broadband luminosities $L(\mathrm{XUV})$ and $L(\mathrm{FUV})$ and the emission line luminosities $L\left(\mathrm{C}_{\mathrm{IV}}\right)$ and $L(\mathrm{Mg}$ II $)$. The figures show a strong correlation between all of these quantities, with Pearson correlation coefficients of $[0.83,0.86,0.87,0.90]$ for the relationships [FUV-C IV, FUV-Mg II, XUV-C IV, XUV$\mathrm{Mg}$ II)], respectively. The probability of a non-correlation is $<3 \times 10^{-3}$ for all four curves. We present quantitative log-log relations $^{25}$ in Table 2. The rms scatter around the [FUV-C IV,

\footnotetext{
${ }^{24}$ The stellar Ly $\alpha$ emission line is the brightest FUV line in M dwarf spectra (France et al. 2013), however, the total emission line flux cannot be retrieved without significant modeling analysis (Wood et al. 2005b; Youngblood et al. 2016).

${ }^{25} \log _{10} L($ band $)=m \times \log _{10} L($ line $)+b$.
}

FUV-Mg II, XUV-C IV, XUV-Mg II)] fit, ( $L$ (band) $-L$ (fit)) $/ L$ (band), is [153\%, 39\%, 109\%, 57\%], respectively.

We have selected $\mathrm{C}_{\mathrm{IV}}$ and $\mathrm{Mg}$ II because they are the two most readily-observable emission lines in the FUV and NUV bandpasses. $\mathrm{Mg}$ II shows a tighter correlation, but as we begin to probe exoplanetary systems at greater distances from the Sun, this relationship will become compromised by the additional contribution from interstellar $\mathrm{Mg}$ II absorption components with radial velocities coincident with the stellar radial velocities (Redfield \& Linsky 2002) as well as the possibility of gas-rich circumstellar environments fueled by mass-loss from short-period gaseous planets (Haswell et al. 2012; Fossati et al. 2015). By contrast, C IV is essentially free from interstellar extinction out to the edge of the Local Bubble where dust opacity begins to contribute appreciably. There are rare exceptions where hot gas in the Local Bubble can contribute small amounts of $\mathrm{C}$ IV attenuation (Welsh et al. 2010), but the effect is considerably less than for $\mathrm{Mg}$ II.

\subsection{Temporally Resolved Spectra and Energetic Flares}

As discussed in the Introduction, temporal variability of the energetic radiation is considerably higher for $\mathrm{M}$ dwarfs than for solar-type stars (Mitra-Kraev et al. 2005), with active M stars showing disk-integrated flux increases of an order of magnitude or more during large flares (Hawley \& Pettersen 1991; Hawley et al. 2003; Osten et al. 2005). The MUSCLES pilot program showed that even inactive $\mathrm{M}$ dwarfs could show impulsive flare behavior in their UV light curves (France et al. 2012a). The UV monitoring component of the MUSCLES Treasury Survey was designed to present a uniform database of flares from exoplanet host stars so that the flare frequency-amplitude relations could be derived for these stars and the impact of impulsive events on the atmospheres or orbiting planets could be assessed. For cases where the X-ray observations were scheduled simultaneously, the overlapping observatory coverage was planned for the HST 5-orbit monitoring campaigns.

The intention of this section is not to give a thorough quantitative description of the flare catalog produced in the survey; that work will be presented in a follow-up paper by R. O. P. Loyd et al. (2016, in preparation). This section is intended to introduce the variability data and present one example of an optically quiet $\mathrm{M}$ dwarf host star that is among the most UV/ $\mathrm{X}$-ray active sources ever observed. Figure 13 shows the light curves of GJ 876, recorded in four bright emission lines, $\mathrm{C}_{\text {II }} \lambda \lambda 1334,1335\left(T_{\text {form }} \sim 3 \times 10^{4} \mathrm{~K}\right), \quad$ Si III $\lambda 1206 \quad\left(T_{\text {form }} \sim\right.$ $\left.4 \times 10^{4} \mathrm{~K}\right), \quad$ Si IV $\lambda \lambda 1394, \quad 1403 \quad\left(T_{\text {form }} \sim 6 \times 10^{4} \mathrm{~K}\right), \quad$ and $\mathrm{N} v \lambda \lambda 1239,1243\left(T_{\text {form }} \sim 2 \times 10^{5} \mathrm{~K}\right)$. The data are binned to a $30 \mathrm{~s}$ cadence and displayed relative to the start time of the first observation. The individual exposures are labeled for reference. Several flares are immediately apparent, the strongest being during the last two orbits of the monitoring campaign. The COS detector background, measured at the same dispersion direction location as the $\mathrm{Si}$ IV lines but offset below the spectral trace in the cross-dispersion direction, is also shown (orange squares) to demonstrate the stability of the instrument during these measurements.

Figure 14 (top) shows a zoom-in on the brightest UV flare, binned to a $10 \mathrm{~s}$ cadence, occurring near $T_{\exp }=17,400 \mathrm{~s}$. Each light curve is normalized to unity during a pre-flare window $(17,100-17,250 \mathrm{~s})$ to enable a comparison of the relative flare responses of each line. The flare/quiescent flux increase in the brightest line, $\mathrm{Si}$ III, is $\sim 110$. C II and $\mathrm{Si}$ IV show flux increases 

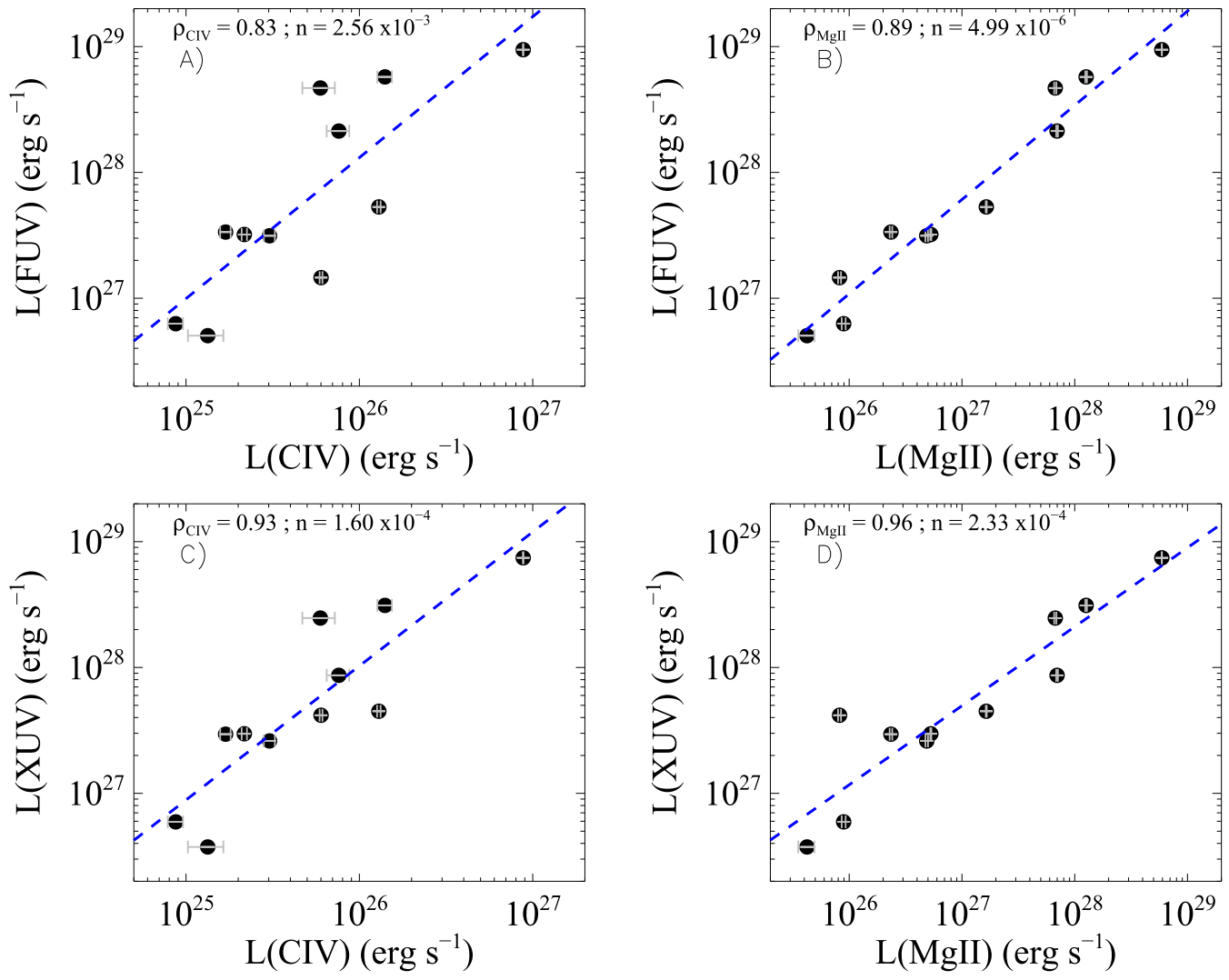

Figure 12. We demonstrate that both $\mathrm{C}$ IV and $\mathrm{Mg}$ II are well correlated with the total FUV and EUV fluxes. (A) $\mathrm{C}$ iv-FUV luminosity relation and corresponding Pearson coefficient and statistical likelihood of a null correlation (top of each panel), (B) Mg II-FUV luminosity relation, (C) C IV-XUV luminosity relation, and (D) Mg II-XUV luminosity relation.

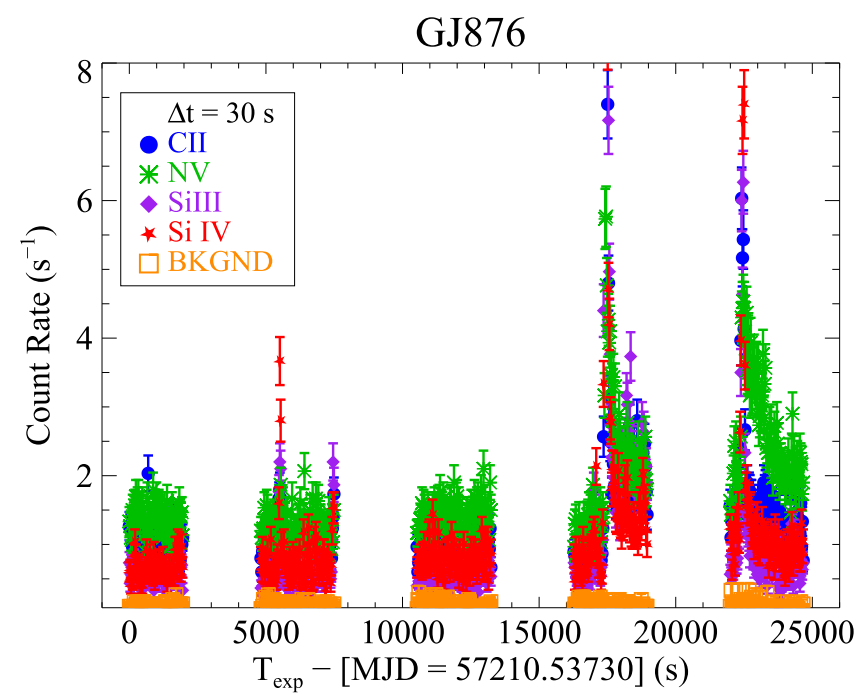

Figure 13. Example of a flare light curve from the five contiguous $H S T$ orbits with the COS G130M mode. The G130M mode provides a wealth of temperature diagnostics from chromosphere and transition region, spanning $\approx 3 \times 10^{4} \mathrm{~K}\left(\mathrm{C}\right.$ II) through $\approx 2 \times 10^{5} \mathrm{~K}(\mathrm{~N}$ v). The instrumental background is shown in orange. The data are displayed at a $30 \mathrm{~s}$ cadence in this figure. The strong flare observed during the fourth orbit is shown in detail in Figure 14.

of the order of $\sim 50$, and $\mathrm{N} v$ and the FUV continuum increase by factors of $\sim 5$. This level of luminosity increase indicates the largest relative UV flare ever directly detected in a diskintegrated observation of a star, despite the relative inactivity of GJ 876 suggested by the Ca II activity index. One observes that the light curves are line-dependent, with intermediate temperature ions showing a larger relative flux increase while the higher temperature ion $(\mathrm{N} v)$ does not respond as strongly but shows a decay time several times longer than $\mathrm{C}$ II, $\mathrm{Si}$ III, and Si IV (Figure 13). Figure 14, (bottom) shows time-resolved line ratios in the GJ 876 light curves. Several emission lines are ratioed to Si IV and normalized to their pre-flare line ratios, and so their relative change is meaningful. In this manner, we can place constraints on the atmospheric temperature regime where most of the observable energy is deposited (our observations do not contain information about energy deposited in cooler or optically thick atmospheric layers). The $\mathrm{Si}$ III/Si IV ratio increases during the flare, while the $\mathrm{C}$ II/Si IV and $\mathrm{N} v / \mathrm{Si}_{\text {IV }}$ ratios, which sample gas both hotter and colder than $\mathrm{Si}$ III, are depressed. This argues that the flare energy distribution is peaked near the $\mathrm{Si}$ III formation temperature, roughly (4-5) $\times 10^{4} \mathrm{~K}$ (with both low and high-energy tails), and evolves during the flare.

In order to understand the impact of these flares on the planets in these systems, we need to convert the observed emission line flares to an estimate of the broadband UV flare energy. Converting the raw spectral light curves into flux units by comparing the orbit-averaged count rates and stellar emission line fluxes, we can create flux-calibrated light curves (Loyd \& France 2014). The total flare energy in a given emission line is then

$$
E(\lambda)=4 \pi d^{2} \int_{t_{\text {start }}}^{t_{\text {end }}} F(\lambda) d t
$$

where $F(\lambda)$ is the line flux in (erg $\mathrm{cm}^{-2} \mathrm{~s}^{-1}$ ), $d$ is the stellar distance, and $t_{\text {start }}$ and $t_{\text {end }}$ are the initial flare rise times and the 

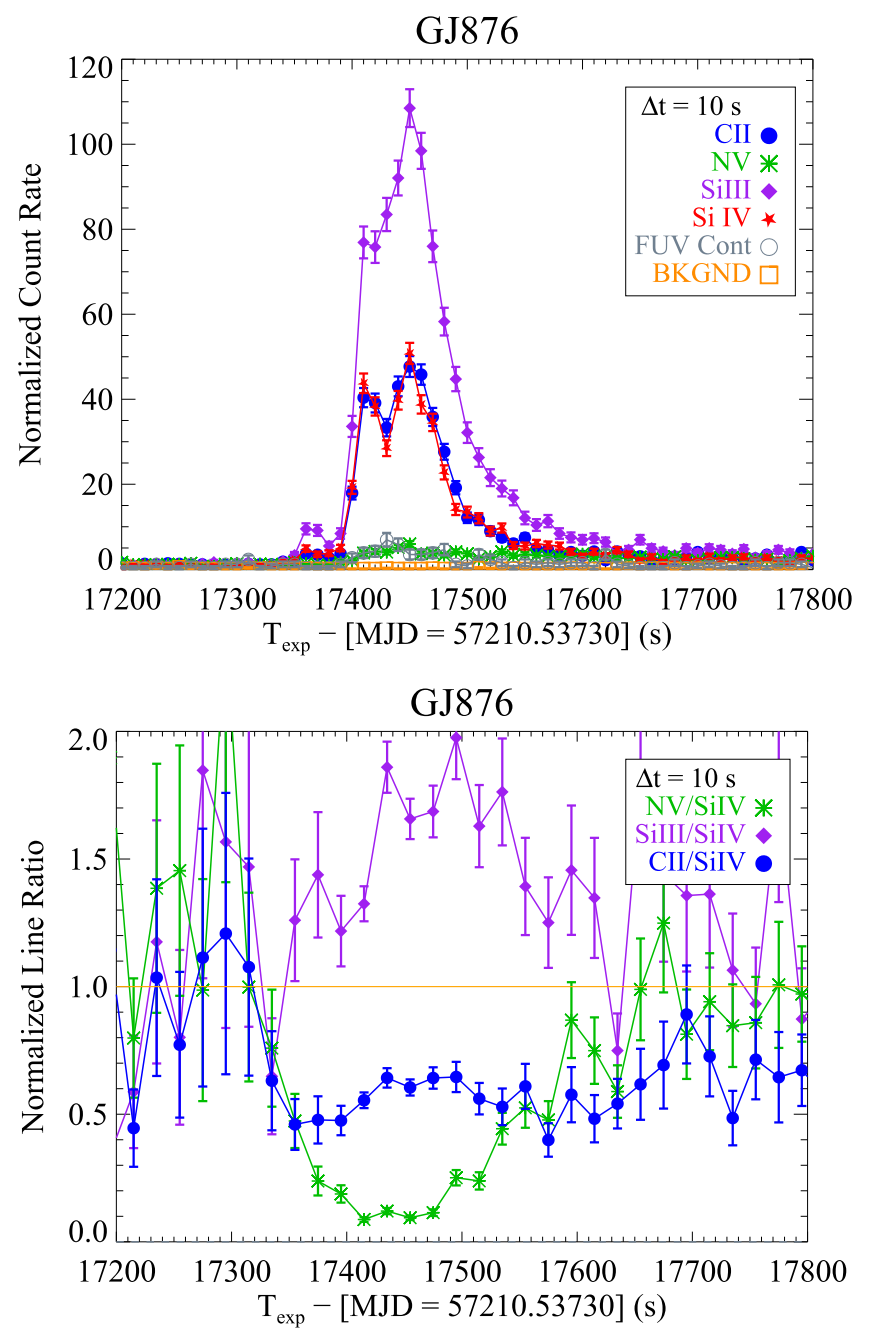

Figure 14. (Top) A zoom-in on the strong UV flare observed on GJ 876 (Figure 13). The peak flare/quiescent flux ratio for this flare in the $\mathrm{Si}^{2+}$ ion is $\approx 110$, making this one of the most extreme disk-integrated UV flares ever detected. The bottom panel shows normalized emission line ratios for several diagnostic lines and their evolution during the flare. This flare peaked in the $\mathrm{Si}$ III ion, with $\mathrm{Si}$ III/Si IV ratios increasing during the flare. Less energy was deposited in the hotter and cooler ions, represented here by the $\mathrm{N} v / \mathrm{Si}$ IVand $\mathrm{C}$ II/Si IVratios, respectively. The full MUSCLES flare catalog will be presented by R. O. P Loyd et al. (2016, in preparation).

time when the flare returns to the quiescent level, respectively. For the bright flare considered here, the exposure ends before the ionic lines return to the quiescent levels, and so we set $t_{\text {end }}$ as the end of the orbit in this case. The Si III and Si IV emission lines are representative of the upper chromosphere and transition region emission formed between $\sim 30$ and $80 \mathrm{kK}$; we use these lines to estimate the total flare emission from these regions. The total energies in these lines are $\log _{10} E$ $(\mathrm{Si}$ III $)=29.17 \mathrm{erg}$ and $\log _{10} E(\mathrm{Si}$ IV $)=29.08 \mathrm{erg}$. We use the $\mathrm{M}$ dwarf stellar atmosphere model of Fontenla et al. (2016) to estimate the total emission in the 300-1700 $\AA$ range that contains the majority of the transition region emission. Emission at $\lambda<300 \AA$ is mainly coronal in origin while emission at $\lambda>1700 \AA$ is mainly cooler chromospheric gas.

The ionized silicon flux energies can be converted to broadband XUV + FUV (300-1700 ^) energy by computing the fractional flux emitted in these lines and the fractional radiative cooling rate that is contributed by the $30-80 \mathrm{kK}$ gas. The total broadband energy is then

$$
\begin{aligned}
& E(300-1700 \AA)=E(\text { Si III }+ \text { Si IV }) \\
& \quad \times\left(f_{300-900}+f_{900-1200}+f_{1200-1700}\right) \times C_{30-80 \mathrm{kK},}
\end{aligned}
$$

where $f_{300-900}$ is the ratio of stellar flux in the 300-900 $\AA$ band to the combined Si III + Si IV flux, $F(300-900 \AA) / F(\mathrm{Si}$ III + Si IV $)$. Similarly, $f_{900-1200}=F(900-1200 \AA) / F($ Si III + Si IV $)$ and $f_{1200-1700}=F(1200-1700 \AA) / F(\operatorname{Si}$ III + Si IV $)$. The $f_{300-900}$, $f_{900-1200}$, and $f_{1200-1700}$ ratios from the model atmosphere of Fontenla et al. (2016) are 32, 14, and 290, respectively. $C_{30-80 \mathrm{kK}}$ is the fraction of the total radiative cooling rate from the upper stellar atmosphere $\left(6000-10^{6} \mathrm{~K}\right)$ contributed by $30-80 \mathrm{kK}$ gas, $C_{30-80 \mathrm{kK}}=\Gamma(30-80 \mathrm{kK}) / \Gamma\left(6000 \mathrm{~K}-10^{6} \mathrm{~K}\right)$, where $\Gamma$ is the radiative cooling rate in units of $\mathrm{erg} \mathrm{cm}^{-3} \mathrm{~s}^{-1}$. Using the cooling rates for this model atmosphere (Fontenla et al. 2016), we compute $C_{30-80 \mathrm{kK}}=16.8 \%$. Note that these are the equilibrium cooling rates for the quiescent model atmosphere, and may be different during the post-reconnection heating associated with the flare. Combining these elements, we estimate that the total UV flare energy associated with this event is $\log _{10} E(300-1700 \AA)=31.18 \mathrm{erg}$, which is comparable to the total quiescent luminosity of the star $E_{\text {flare }}(\mathrm{UV}) \sim 0.3 L_{*} \Delta t(\Delta t=1 \mathrm{~s})$.

In a future work, R. O. P. Loyd et al. (2016, in preparation) will show that HST monitoring observations are able to measure and quantify the $\mathrm{M}$ dwarf flare amplitude-frequency distribution. Critically, HST provides the sensitivity and spectral resolution to analyze light curves covering a range of formation temperatures, and capture the short duration events that dominate the flare distribution. A dedicated HST spectroscopic flare monitoring program is currently the best avenue for understanding the energy and temporal distribution of flares and their potential influence on low-mass planets.

\subsubsection{X-Ray Light Curves}

When possible, X-ray observations were taken in concert with the UV light curves described here. However, this was only possible for about half of the MUSCLES observations. Figure 15 shows the non-simultaneous X-ray light curve of GJ $876,{ }^{26}$ finding another large flare on this object. The X-ray spectra of both the quiescent and flare periods have been fitted, and the overall X-ray luminosity increased by a factor of $\sim 10$ during the flare. The actual luminosity increase was likely larger but diluted by the cadence adopted to provide sufficient $\mathrm{S} / \mathrm{N}$ in each time bin. Given the similarity of the UV and X-ray light curves of dMe stars observed by XMM (Mitra-Kraev et al. 2005), it seems likely that these large flares are similar events and occur with regularity on GJ 876. We note that Poppenhaeger et al. (2010) also noted a high level of X-ray activity on GJ 876 and France et al. (2012a) observed a large UV flare in the very first MUSCLES observations. This apparently inactive star displays a flare outburst almost every time it is observed at wavelengths below the atmospheric cut-

\footnotetext{
26 This observation was scheduled simultaneously, but HST entered safe mode during the scheduled period during early 2015 June and the Chandra observations executed without $H S T$. The HST observations were rescheduled about a month later, without supporting X-ray observations.
} 


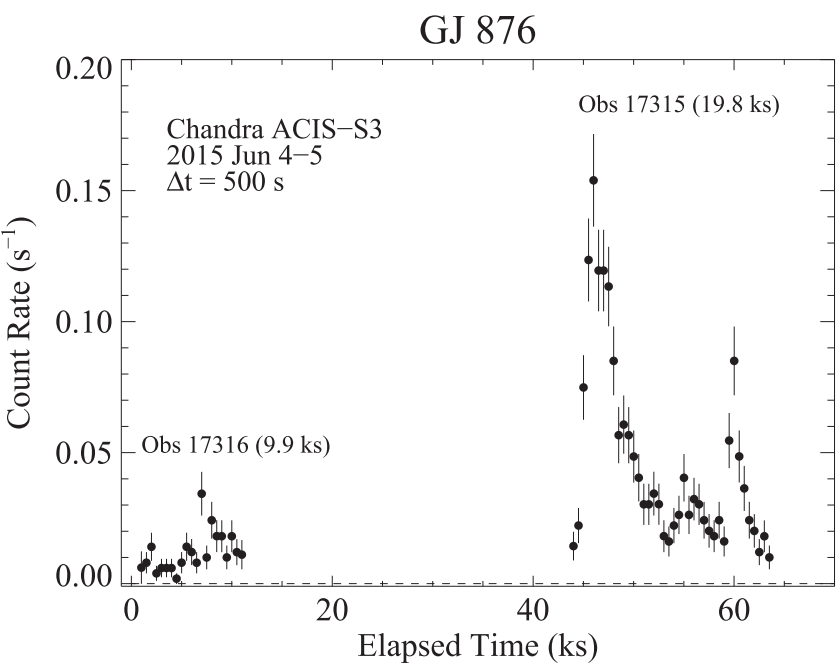

Figure 15. Example X-ray flare light curve of GJ 876 from the Chandra program to support the five contiguous monitoring orbits carried out with COS G130M. An HST safing event prevented this light curve from being simultaneous with the FUV light curves shown in Figures 13 and 14.

off. While we have elected to focus on the most UV-active star in the MUSCLES data set, roughly half of our targets displayed UV flare activity. This will be explored in greater detail in R. O. P. Loyd et al. (2016, in preparation).

\section{DISCUSSION}

\subsection{Chromospheric and Transition Region Activity of GJ 1214: On, Off, or Variable?}

We use GJ 1214, the only star in our sample with an effective temperature of $<3000 \mathrm{~K}$, to investigate the evolution of upper atmosphere activity toward the stellar/sub-stellar boundary. In the MUSCLES pilot study, we presented an upper limit to the Ly $\alpha$ flux of GJ 1214 based on earlier STIS G140M observations. GJ 1214 has a spectral type of M4.5 V with $T_{\text {eff }}=2949 \pm 30 \mathrm{~K}$ (Kundurthy et al. 2011; consistent with the $2935 \mathrm{~K}$ derived from the photospheric model fitting described in Paper III). It was the most distant source in the MUSCLES pilot study and the only star for which $\mathrm{H}_{\text {I }} \mathrm{Ly} \alpha$ was not detected. This non-detection was approximately a factor of 10 below the expected flux level based on an extrapolation of the $F(\operatorname{Ly} \alpha)$ versus $F(\mathrm{Mg}$ II $)$ relation (France et al. 2013) and was made more surprising by the solid detection of C IV $\lambda 1548$ emission. This led us to speculate about the possibility of a high molecular fraction atmosphere that suppresses atomic hydrogen emission, or possibly an "on/off" behavior where the basal flux level is very low and the chromospheric and coronal emission can only be observed during flares (as has been noted for the M8 star VB 10 by Linsky et al. 1995; however, more recent observations demonstrated an "always on" behavior, Hawley \& Johns-Krull 2003). Subsequently, X-rays from GJ 1214 were detected by XMM-Newton (Lalitha et al. 2014). All of the source counts during the $X M M$ observations occurred in a single time bin, suggesting that the "on/off" scenario may be correct.

The new MUSCLES observations instead argue for a weak but persistent high-energy flux from GJ 1214. We have detected the full complement of FUV and NUV emission lines from GJ 1214 with the deeper MUSCLES treasury data (including direct observation of $\operatorname{Ly} \alpha$, see Paper II; Table 3).
While the Ly $\alpha$ reconstruction is uncertain due to the low $\mathrm{S} / \mathrm{N}$ and the small fraction of the line profile that is detected, we can compare the observed flux from the star over multiple epochs. The Ly $\alpha$ (STIS G140M) and C IV + Mg II (COS G160M + STIS G230L) observations in France et al. (2013) were separated by roughly 15 months, while the 2015 MUSCLES Treasury data were obtained over a period of $<2$ days. We find that the observed flux level from GJ 1214 in 2015, $F_{2015}(\mathrm{Ly} \alpha)=1.8( \pm 0.3) \times 10^{-15} \mathrm{erg} \mathrm{cm}^{-2} \mathrm{~s}^{-1}$, is consistent with the upper limit presented previously, $F_{2013}(\operatorname{Ly} \alpha) \leqslant$ $2.4 \times 10^{-15} \mathrm{erg} \mathrm{cm}^{-2} \mathrm{~s}^{-1}$. The reconstructed flux is approximately twice this value, $F_{2015 \text {,recon }(\operatorname{Ly} \alpha)}=5.5 \times$ $10^{-15} \mathrm{erg} \mathrm{cm}^{-2} \mathrm{~s}^{-1}$, which is still roughly a factor of three lower than the expected reconstructed Ly $\alpha$ flux based on the $F$ (Ly $\alpha$ ) versus $F(\mathrm{Mg}$ II) relation (Youngblood et al. 2016). We find that the total $\mathrm{C}$ IV brightness has increased by a factor of two compared to our earlier study $\left(F_{2015}(\mathrm{C}\right.$ IV $)=5.2$ $( \pm 1.2) \times 10^{-16} \mathrm{erg} \mathrm{cm}^{-2} \mathrm{~s}^{-1}$ versus $F_{2013}\left(\mathrm{C}_{\mathrm{IV}}\right)=2.6( \pm 0.5) \times$ $\left.10^{-16} \mathrm{erg} \mathrm{cm}^{-2} \mathrm{~s}^{-1}\right)$, while the $\mathrm{Mg}$ II flux is approximately constant $\left(F_{2015}(\mathrm{Mg} \mathrm{II})=1.7( \pm 0.3) \times 10^{-15} \mathrm{erg} \mathrm{cm}^{-2} \mathrm{~s}^{-1}\right.$ versus $F_{2013}(\mathrm{Mg}$ II $\left.)=2.2( \pm 0.2) \times 10^{-16} \mathrm{erg} \mathrm{cm}^{-2} \mathrm{~s}^{-1}\right)$.

GJ 1214 displays fractional luminosities that are typical for the mid/late $M$ dwarfs in the MUSCLES survey (Table 3; Figures 10 and 11). It has the lowest absolute levels of highenergy radiation (Figure 8) due to its low mass. The low intrinsic luminosity level and the star's relatively large distance $(d=13 \mathrm{pc})$ can explain the non-detection of $\mathrm{Ly} \alpha$ in the previous work. It appears that GJ 1214 is simply a scaled-down version of the typical planet-hosting mid-M dwarf, and is not in a fundamentally different state of chromospheric activity. While GJ 1214 clearly has flares, we have now shown that the basal flux level of this star is similar to other low-mass exoplanet host stars. Combining this result with the high activity levels on M5 stars such as GJ 876, we conclude that special time-dependent photochemistry (other than the incorporation of impulsive flares and longer-term variability; Section 1) is not necessary down to spectral type M5.

\subsection{Comparison of UV Observations to Coronal Models}

The MUSCLES panchromatic SED creation relies on direct observation at all wavelengths, except in the red/IR wavelengths $(\lambda>6000 \AA)$ and the XUV/FUV region between 50 and $1170 \AA$. As discussed in the Introduction, the EUV regulates heating and mass-loss in planetary atmosphere, particularly for short-period planets around stars with large EUV and X-ray fluxes. However, obtaining a complete EUV spectrum of any cool star other than the Sun is currently impossible due to attenuation by the ISM. Our approach to filling in this observationally inaccessible gap is to employ a coronal model from 50 to $100 \AA$ (a single or two-temperature APEC model, Smith et al. 2001) and models of solar active regions from 100 to $1170 \AA$ (Fontenla et al. 2011; Linsky et al. 2014). This approach assumes a scaling between the chromospheric Ly $\alpha$ emission and the chromospheric + transition region + coronal flux that contributes to the EUV bands, particularly between 100 and $400 \AA$.

An alternative approach has been taken by Sanz-Forcada et al. (2011), who used X-ray observations of the coronae of exoplanet host stars and an emission measure distribution technique to predict the EUV and part of the FUV spectra of low-mass host stars. The synthetic spectral output of coronal models for three of the MUSCLES stars ( $\epsilon$ Eri, GJ 436, and GJ 876) are available on 
Table 3

MUSCLES Broadband and Emission Line Luminosity ${ }^{\mathrm{a}, \mathrm{b}, \mathrm{c}}$

\begin{tabular}{|c|c|c|c|c|c|c|c|c|}
\hline Target & $L_{\mathrm{Bol}}$ & $f(\mathrm{XUV})$ & $f(\mathrm{FUV})$ & $f(\mathrm{NUV})$ & $f(\operatorname{Ly} \alpha)$ & $f(\mathrm{~N} v)$ & $f(\mathrm{C}$ IV $)$ & $f(\mathrm{Mg}$ II $)$ \\
\hline GJ 1214 & 31.15 & -4.50 & -4.45 & -4.20 & -4.52 & -6.48 & -6.03 & -5.53 \\
\hline GJ 176 & 32.12 & -4.46 & -4.40 & -3.81 & -4.51 & -6.61 & -6.01 & -4.91 \\
\hline GJ 436 & 32.02 & -4.41 & -4.52 & -4.02 & -4.59 & -6.93 & -6.54 & -5.33 \\
\hline GJ 581 & 31.64 & -4.50 & -4.84 & -4.18 & -4.90 & -7.23 & -6.70 & -5.69 \\
\hline GJ 667C & 31.70 & -4.21 & -4.17 & -3.86 & -4.23 & -7.11 & -6.47 & -5.33 \\
\hline GJ 832 & 31.79 & -4.30 & -4.29 & -3.60 & -4.36 & -6.80 & -6.46 & -5.07 \\
\hline GJ 876 & 31.69 & -4.04 & -4.53 & -4.33 & -4.68 & -6.24 & -5.91 & -5.77 \\
\hline HD 40307 & 32.98 & -4.57 & -4.31 & -2.41 & -4.37 & -8.20 & -7.21 & -5.15 \\
\hline HD 85512 & 32.78 & -4.82 & -4.45 & -3.01 & -4.51 & -7.79 & -6.89 & -4.93 \\
\hline HD 97658 & 33.12 & -4.61 & -4.36 & -2.05 & -4.43 & -7.86 & -6.97 & -5.02 \\
\hline$\epsilon$ Eri & 33.08 & -4.15 & -4.10 & -1.97 & -4.20 & -6.97 & -6.13 & -4.30 \\
\hline
\end{tabular}

Notes.

a Flux measurements are averaged over all exposure times for individual observations, and broadband SEDs are constructed as described in Loyd et al. (2016).

${ }^{\mathrm{b}}$ All quantities presented as $\log _{10}\left(L_{\mathrm{Bol}}\right)$ or $f($ band $)=\log _{10}\left(L(\right.$ band $\left.) / L_{\mathrm{Bol}}\right)$.

${ }^{\mathrm{c}}$ Broadband bandpasses are defined as Bol $\Delta \lambda=5 \AA-\infty$, XUV $\Delta \lambda=5-911 \AA$, FUV $\Delta \lambda=912-1700 \AA$ (including Ly $\alpha$ ), NUV $\Delta \lambda=1700-3200 \AA$.

the X-exoplanets website, ${ }^{27}$ and provide overlap with the high S/N MUSCLES data in the 1150-1200 $\mathrm{A}$ region. We downloaded these synthetic spectra to compare the coronal model fluxes with the data for the bright $\mathrm{C}$ III $\lambda 1175$ multiplet. These lines are formed in the transition region at $T_{\text {form }} \sim 6 \times 10^{4} \mathrm{~K}$, and therefore provide a good diagnostic for how well coronal models are able to reproduce the UV emission throughout the FUV. The $X$-exoplanets synthetic spectra are provided in units of photons s $\mathrm{s}^{-1} \mathrm{~cm}^{-2}$ bin $^{-1}$ with a variable bin size. The spectra are multiplied by a factor of (bin size) $)^{-1}$ and then integrated over wavelength to compute the integrated $\mathrm{C}$ III line photons $\mathrm{s}^{-1} \mathrm{~cm}^{-2}$. The MUSCLES data were converted to photons and integrated over the same wavelength interval (1174-1177 $\mathrm{A}$ ).

We find that the coronal models systematically underpredict the UV emission line strengths by factors of a few to tens (Table 4). For $\epsilon$ Eri, we find $F_{\text {data }}(\mathrm{C}$ III $)=1.8 \times 10^{-2}$ photons s ${ }^{-1} \mathrm{~cm}^{-2}$ versus $F_{\text {Xexoplanets }}\left(\mathrm{C}_{\mathrm{III}}\right)=6.6 \times 10^{-4}$ photons s $\mathrm{s}^{-1} \mathrm{~cm}^{-2}$. For GJ 436, we find $F_{\text {data }}\left(\mathrm{C}\right.$ III) $=4.2 \times 10^{-5}$ photons s $\mathrm{s}^{-1} \mathrm{~cm}^{-2}$ versus $F_{\text {Xexoplanets }}\left(\mathrm{C}_{\text {III }}\right)=8.0 \times 10^{-6}$ photons s $\mathrm{sm}^{-1} \mathrm{~cm}^{-2}$. For GJ 876 , we find $\quad F_{\text {data }}\left(\mathrm{C}\right.$ III) $=6.5 \times 10^{-4}$ photons s $\mathrm{s}^{-1} \mathrm{~cm}^{-2} \quad$ versus $F_{\text {Xexoplanets }}(\mathrm{C}$ III $)=1.9 \times 10^{-5}$ photons s ${ }^{-1} \mathrm{~cm}^{-2}$. The coronal models underpredict these transition region fluxes by factors of approximately 27, 5, and 33 for $\epsilon$ Eri, GJ 436, and GJ 876, respectively. This difference underscores the importance of having empirical inputs for transition region and chromospheric emission in the calculations of the EUV flux from cool stars (see also the discussion presented in Linsky et al. 2014). The systematic underprediction of the transition region emission brings into question the accuracy of the coronal models in the 400-900 EUV region which is dominated by chromospheric and transition region line and continuum spectra. Another important factor is the time variability in the coronal and chromospheric emission from these stars, which may not be the same. The MUSCLES database provides an excellent resource for emission measure distributionbased stellar atmosphere models that simultaneously take into account both the intermediate- and high-temperature regions of the stellar atmosphere (e.g., Chadney et al. 2015) and may provide more accurate EUV flux estimates for the exoplanet community.

\footnotetext{
${ }^{27}$ http://sdc.cab.inta-csic.es/xexoplanets/jsp/homepage.jsp
}

Table 4

Comparison of MUSCLES C III $\lambda 1175$ Emission and X-exoplanets Spectral Synthesis Prediction

\begin{tabular}{lcc}
\hline \hline Star & $F(\mathrm{C}$ III), MUSCLES & $F(\mathrm{C}$ III), X-exoplanets \\
\hline GJ 876 & $6.5 \times 10^{-4}$ & $1.9 \times 10^{-5}$ \\
GJ 436 & $4.2 \times 10^{-5}$ & $8.0 \times 10^{-6}$ \\
$\epsilon$ Eri & $1.8 \times 10^{-2}$ & $6.6 \times 10^{-4}$ \\
\hline
\end{tabular}

Note.

${ }^{\mathrm{a}}$ All fluxes in units of (photons $\mathrm{s}^{-1} \mathrm{~cm}^{-2}$ ).

\subsection{Star-Planet Interactions Observed in Transition Region Emission Lines}

It has been suggested that stellar and exoplanetary magnetic fields can interact in exoplanetary systems (Shkolnik et al. 2003; Lanza 2008), possibly manifesting as enhanced stellar activity relative to nominal age-rotation-activity relationships for isolated main-sequence stars (Barnes 2007; Mamajek \& Hillenbrand 2008). The magnitude of this interaction, as measured by the energy dissipated in the stellar atmosphere, may be proportional to the strength of the stellar magnetic field, the planetary magnetic field, and the relative speed of the planet's orbital velocity compared to the stellar magnetic rotation rate. While the stellar magnetic field and orbital velocity can be readily measured, exoplanet magnetic fields have proven notoriously hard to detect (see, e.g., Lecavelier des Etangs et al. 2011 and Hallinan et al. 2013), with very few possible detections from low-frequency radio observations (Lecavelier des Etangs et al. 2013) and early-ingress measurements of NUV and optical transit light curves (Fossati et al. 2010; Lai et al. 2010; Vidotto et al. 2010; Llama et al. 2011; Cauley et al. 2015). If the above dependencies on the magnetic field and velocities are valid, then a general trend of enhanced energy dissipation, which could be observed as enhanced emission from the stellar corona (X-rays; Kashyap et al. 2008) or chromosphere (Ca II; Shkolnik et al. 2005), should correlate with $M_{\text {plan }} / a_{\text {plan }}$, where $M_{\text {plan }}$ is the planetary mass and $a_{\text {plan }}$ is the semimajor axis. Because magnetic field strength increases with planetary mass in the solar system, one would expect that the most massive, closest-in planets in exoplanetary systems should produce the largest signal on their host stars. However, almost every claimed detection of a 


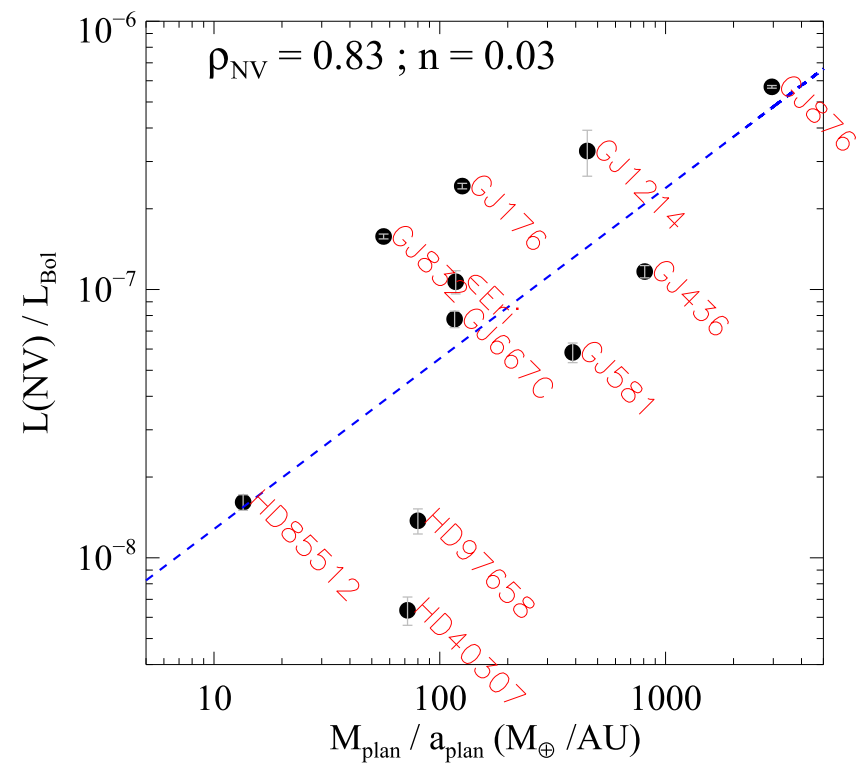

Figure 16. Fractional N v luminosity is positively correlated with a measure of the "star-planet interaction strength," $M_{\text {plan }} / a_{\text {plan }}$ (Shkolnik 2013), where $M_{\text {plan }}$ is the mass of the most massive planet in the system (in Earth masses) and $a_{\text {plan }}$ is the semimajor axis of the most massive planet in the system (in au). The Pearson coefficient and statistical likelihood of a null correlation is shown at the top. This provides tentative evidence that the presence of short-period planets enhances the transition region activity on low-mass stars, possibly through the interaction of their magnetospheres.

modulation in activity on exoplanet host stars or connection with orbital phase has not been confirmed by follow up observation or re-analysis of the same data set (e.g., Shkolnik et al. 2008; Poppenhaeger et al. 2010).

The search for star-planet interaction (SPI) has predominantly focused on hot Jupiters orbiting F, G, and K stars. The MUSCLES database allows us to examine potential star-planet interactions for a range of planetary masses and semimajor axes on a new class of optically inactive low-mass star. Furthermore, the MUSCLES database allows us to explore SPI as a function of emission line formation temperature, which can constrain the possible location of magnetic field line connection in the stellar atmosphere and the subsequent location of plasma heating. Figure 16 shows a correlation between the $\mathrm{Nv}$ fractional luminosity and $M_{\text {plan }} / a_{\text {plan. }}$. The $\mathrm{N} \mathrm{v}-M_{\text {plan }} / a_{\text {plan }}$ relation has a Pearson correlation coefficient of 0.83 and a statistical noncorrelation likelihood of 0.035 . This suggests that the systems with close-in, massive planets may indeed be generating enhanced transition region activity, as probed by this $\sim 2 \times 10^{5}$ $\mathrm{K}$ gas. ${ }^{28}$ On the other hand, we do not observe a correlation with the lower-temperature chromospheric gas traced by $\mathrm{Mg}$ II $\left(T_{\text {form }} \sim(1-2) \times 10^{4} \mathrm{~K}\right)$. The $\mathrm{Mg}$ II $-M_{\text {plan }} / a_{\text {plan }}$ relation, for instance, displays a correlation coefficient and probability of a null correlation, -0.3 and 0.06 , respectively. Figure 17 presents the Pearson correlation coefficients for the relation between the fractional luminosity and $M_{\text {plan }} / a_{\text {plan }}$ for several lines in the MUSCLES spectra. While the null-hypothesis cannot be ruled out with high confidence for individual hot gas lines (i.e., the $n$ values are not very low), the trend with line formation temperature suggests that the connection between

\footnotetext{
${ }^{28}$ For systems with additional, lower-mass planets, we also computed the correlation between $L$ (line) $/ L_{\mathrm{Bol}}$ and the summed SPI integration strength for all $j$ planets in the system, $\Sigma_{j}\left(M_{j} / a_{j}\right)$, and find that the correlation is somewhat weakened, Pearson coefficient $=0.82$ and $n=0.2$.
}

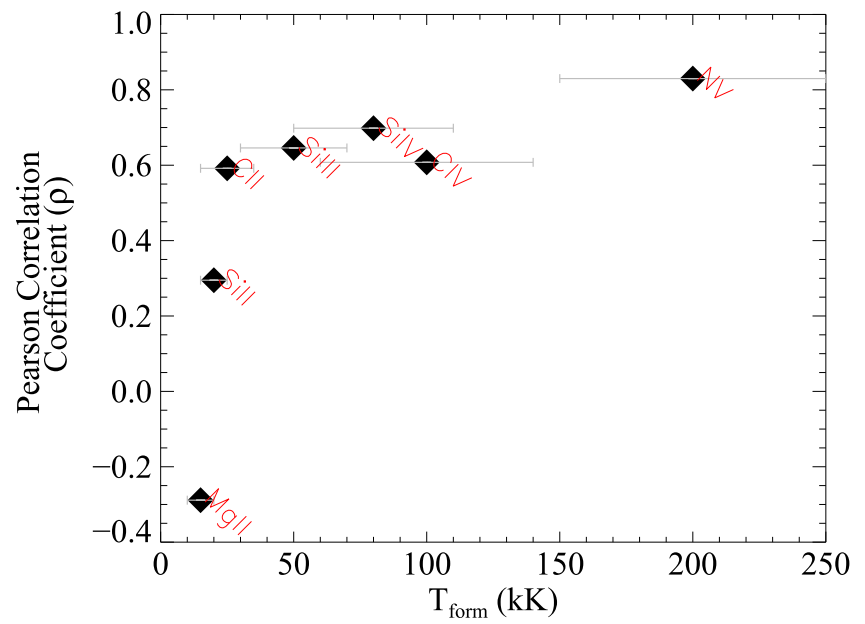

Figure 17. Pearson correlation coefficients characterizing the strength of the relationship between the fractional emission luminosity in strong chromospheric and transition region lines and $M_{\text {plan }} / a_{\text {plan. }}$. These are plotted as a function of formation temperature to better understand where the SPI process is depositing energy into the stellar atmosphere. Specific transitions are labeled in red, and the range of formation temperatures (Fontenla et al. 2016) are shown as the gray error bars. We observe no significant correlation for formation temperatures $<5 \times 10^{4} \mathrm{~K}$, while the highest formation temperatures show the strongest correlations. This suggests that the SPI deposits significantly more energy in the transition region than in the chromosphere for M stars.

hot gas emission enhancement and $M_{\text {plan }} / a_{\text {plan }}$ may be real. This suggests that the SPI correlation would not be observed in $\mathrm{Ca}$ II, which has a formation temperature distribution comparable to $\mathrm{Mg}$ Iin $\mathrm{M}$ dwarfs (Fontenla et al. 2016).

The correlation of the hot gas lines in the MUSCLES sample is somewhat surprising given the null result found by Shkolnik (2013) for GALEX fluxes. As the GALEX observations are broader band measurements, there is an uncertain and variable contribution from the stellar photosphere that must be subtracted, lines from a range of formation temperatures are simultaneously included, and the highest temperature lines (e.g., Nv) are excluded. Therefore, potential SPI signals are significantly diluted in UV imaging surveys relative to the MUSCLES survey, which focuses on lower-mass stars with less continuum emission and spectrally resolved line profiles that can be robustly measured. This result argues that very low spectral resolution $(R<500)$ observations at FUV or NUV wavelengths are not a promising avenue for discovering SPIdriven activity enhancements in M dwarfs.

We emphasize that given the small sample size, the detection of SPI in the MUSCLES sample should be viewed with caution. One explanation for these results is a simple scaling with stellar effective temperature. Our inactive $\mathrm{K}$ dwarfs have low fractional luminosities and low-mass planetary systems, driving one end of the correlations in some cases. A larger sample that is better controlled for systematics is needed to confirm the results presented here. A future spectroscopic survey of $\mathrm{G}, \mathrm{K}$, and $\mathrm{M}$ exoplanet host stars with the COS G130M mode (to cover $\mathrm{Nv}, \mathrm{Si}$ IV, and $\mathrm{C}_{\text {II }}$ ) could be a promising data set with which to characterize enhanced magnetospheric stellar emission generated by SPI.

\section{SUMMARY}

We have presented the first panchromatic survey of $\mathrm{M}$ and $\mathrm{K}$ dwarf exoplanet host stars from X-ray to UV to optical to IR wavelengths. The MUSCLES Treasury Survey is built upon 
contemporaneous Chandra (or XMM), HST, and ground-based data. The $5 \AA-5 \mu \mathrm{m}$ SEDs have been assembled and hosted as high-level science products on the MAST website. The main purpose of this paper was to present the motivation and overview of the MUSCLES Treasury Survey; more detailed analyses of the spectrally and temporally resolved SEDs, their impact on atmospheric photochemistry, and the suitability of $\mathrm{M}$ dwarfs as habitable planet hosts will be addressed by our team in future publications.

The main results of this work are the following.

1. All stars show energetic radiation (X-ray through UV) at all times during the observations. Chromospheric, transition region, and coronal emission is directly observed from all targets. Despite all but one of our targets having $\mathrm{H} \alpha$ in absorption (that is, "inactive" in the traditional optical sense), all of the MUSCLES stars are X-ray and UV active.

2. We have provided empirically derived relations to compute the FUV and XUV stellar luminosity from $\mathrm{Mg}$ II and $\mathrm{C}$ IV emission line fluxes.

3. The FUV/NUV flux ratio, an indicator for the potential abiotic formation of $\mathrm{O}_{2}$ and $\mathrm{O}_{3}$, declines with increasing stellar effective temperature by more than two orders of magnitude from $T_{\text {eff }}=3000-5000 \mathrm{~K}$. Consequently, the FUV/NUV flux ratio declines by more than two orders of magnitude as $\mathrm{HZ}$ orbital distances increase from 0.1 to $0.7 \mathrm{au}$. The total FUV radiation field strength increases by factors of 2-3 over this distance, while the XUV radiation field strength is approximately constant. The average FUV and XUV fluxes in the HZs of all $\mathrm{K}$ and $\mathrm{M}$ dwarfs studied are $\approx 10-70 \mathrm{erg} \mathrm{cm}^{-2} \mathrm{~s}^{-1}$. The SED of the radiation field changes dramatically for different $\mathrm{HZ}$ distances around low-mass stars, but the intensity of the high-energy radiation field does not.

4. Despite their weak optical activity indicators (e.g., Ca II emission core equivalent widths), several of our stars display extremely strong UV and X-ray flares. Flare/ quiescent flare increases by a factor of $\sim 10$ are common on at least half of our stars with the strongest flares showing $E_{\text {flare }}(\mathrm{UV}) \sim 0.3 L_{*} \Delta t$.

5. Emission measure distribution models based on X-ray (coronal) data alone underestimate the FUV transition region flux by factors of $\sim 5-30$, meaning that these models should not be used for calculating the FUV radiation field of exoplanet host stars. The MUSCLES database provides an excellent resource for emission measure modeling of the stellar atmosphere where lines formed in the chromosphere, transition region, and corona can all be taken into account.

6. We present tentative evidence for star-planet interaction by measuring the fractional emission line luminosity as a function of the star-planet interaction strength, $M_{\text {plan }} /$ $a_{\text {plan }}$. Only the high-temperature transition region lines ( $\mathrm{C}$ IV and $\mathrm{N} v$ ) show a positive correlation. No correlation exists for lines with formation temperatures below $10^{5} \mathrm{~K}$, suggesting that the interaction takes place primarily in the transition region or corona. Moderate-resolution FUV spectroscopy appears to be a promising avenue to further characterize star-planet interactions, while narrow-band FUV and NUV imaging are of less utility for characterizing the interactions of stars and planets due to bandpass and spectral resolution limitations.
The data presented here were obtained as part of the HST Guest Observing programs \#12464 and \#13650 as well as the COS Science Team Guaranteed Time programs \#12034 and \#12035. This work was supported by STScI grants HST-GO12464.01 and HST-GO-13650.01 to the University of Colorado at Boulder. Data for the MUSCLES Treasury Survey were also acquired as part of Chandra and XMM guest observing programs, supported by Chandra grants GO4-15014X and GO5-16155X from the Smithsonian Astrophysical Observatory and NASA $X M M$ grant NNX16AC09G to the University of Colorado at Boulder. This work is based in part upon observations obtained with the Apache Point Observatory $3.5 \mathrm{~m}$ and $0.5 \mathrm{~m}$ telescopes, which are owned and operated by the Astrophysical Research Consortium. K.F. thanks Evgenya Shkolnik for enjoyable discussions about low-mass stars and Jorge Sanz-Forcada for assistance with the absolute flux levels of the X-exoplanets model spectra. The MUSCLES team also thanks STScI program coordinator Amber Armstrong for her long hours spent scheduling these complicated coordinated observations. P.C.S. gratefully acknowledges an ESA Research Fellowship. S.L.H. acknowledges support from NSF grant AST 13-11678. F.T. is supported by the National Natural Science Foundation of China (41175039), the Startup Fund of the Ministry of Education of China (20131029170), and the Tsinghua University Initiative Scientific Research Program.

\section{APPENDIX MUSCLES TARGETS}

GJ 1214-GJ 1214 is a late M dwarf (M4.5 V) at a distance of $14.6 \mathrm{pc}$, making it the coolest and most distant $\mathrm{M}$ dwarf in the MUSCLES Treasury Survey. It has an age of $6 \pm 3 \mathrm{Gyr}$ (Charbonneau et al. 2009), a roughly solar metallicity ([Fe/ $\mathrm{H}]=+0.05$; Neves et al. 2014), and shows signs of optical flare activity (Kundurthy et al. 2011). Coronal emission from GJ 1214 was recently observed by XMM-Newton (Lalitha et al. 2014) with $\log _{10} L_{X}=25.87 \mathrm{erg} \mathrm{s}^{-1}$. GJ $1214 \mathrm{~b}$ is a transiting super-Earth $\left(M_{\text {plan }} \approx 6.5 M_{\oplus}, a_{\text {plan }}=0.014 \mathrm{au}\right.$; Charbonneau et al. 2009), possibly harboring a dense, water-rich (Bean et al. 2010; Désert et al. 2011), and likely cloudy (Kreidberg et al. 2014) atmosphere.

GJ 876-GJ 876 is an M5 dwarf at a distance of 4.7 pc. GJ 876 has super-solar metallicity $([\mathrm{Fe} / \mathrm{H}]=0.14$; Neves et al. 2014); differing estimates on the stellar rotation period $\left(40 \leqslant P_{*} \lesssim 97\right.$ days) result in large uncertainties in the age estimate for this system, 0.1-5 Gyr (Rivera et al. 2005, 2010; Correia et al. 2010). While the star would be characterized as weakly active based on its $\mathrm{H} \alpha$ absorption spectrum, UV and X-ray observations show the presence of an active upper atmosphere (Walkowicz et al. 2008; France et al. 2012a; $\log _{10} L_{X}=26.48 \mathrm{erg} \mathrm{s}^{-1}$; Poppenhaeger et al. 2010). GJ 876 has a rich planetary system with four planets ranging from a super-Earth (GJ $876 \mathrm{~d}, M_{\text {plan }} \approx 6.6 M_{\oplus}$ ) in a short-period orbit ( $a_{\text {plan }}=0.02 \mathrm{au}$; Rivera et al. 2010) to two Jovian-mass planets in the HZ (GJ 876b, $M_{\text {plan }} \approx 2.27 M_{\text {Jup }}, a_{\text {plan }}=0.21$ au; GJ $876 \mathrm{c}, M_{\text {plan }} \approx 0.72 M_{\text {Jup }}, a_{\text {plan }}=0.13$ au; Rivera et al. 2010).

GJ 581-GJ 581 is an M5 dwarf at a distance of 6.3 pc. It is estimated to have an age of $8 \pm 1 \mathrm{Gyr}$ (Selsis et al. 2007) and a somewhat subsolar metallicity, $[\mathrm{Fe} / \mathrm{H}]=-0.20$ (Neves et al. 2014). GJ 581 was not detected in early X-ray surveys $\left(\log _{10} L_{X}<26.89 \mathrm{erg} \mathrm{s}^{-1}\right.$; Poppenhaeger et al. 2010), however, $X$-rays were subsequently detected, $\log _{10} L_{X}=26.11 \mathrm{erg} \mathrm{s}^{-1}$, by Swift (Vitale \& France 2013). Its optical spectrum displays 
$\mathrm{H} \alpha$ in absorption, and therefore chromospheric and coronal activity are thought to be low for this target. GJ 581 has one of the richest known planetary systems, with possibly up to six planets (four confirmed) including several with Earth/superEarth masses (Mayor et al. 2009; Tuomi 2011). GJ $581 \mathrm{~d}$ is a super-Earth $\left(M_{\text {plan }} \approx 6 M_{\oplus}\right)$ that resides on the outer edge of the HZ $\left(a_{\text {plan }}=0.22 \mathrm{au}\right.$; von Braun et al. 2011; Wordsworth et al. 2011).

GJ 176-GJ 176 is an M2.5 dwarf at a distance of $9.4 \mathrm{pc}$. It is estimated to have an age between 0.56 and $3.62 \mathrm{Gyr}$ (Kiraga \& Stepien 2007; Sanz-Forcada et al. 2011) and a solar metallicity of $[\mathrm{Fe} / \mathrm{H}]=-0.01$ (Neves et al. 2014). GJ 176 has an archival $\mathrm{X}$-ray luminosity of $\left(\log _{10} L_{X}=27.48 \mathrm{erg} \mathrm{s}^{-1}\right.$; Poppenhaeger et al. 2010) and a 39 day rotational period (Robertson et al. 2015). GJ $176 \mathrm{~b}$ was initially detected as a $24 M_{\oplus}$ planet in a 10 day orbit (Endl et al. 2008), but subsequent analysis of the stellar light curve has refined this estimate to a super-Earth mass planet $\left(8.3 M_{\oplus}\right)$ in a 6.6 day orbit (Butler et al. 2009; Forveille et al. 2009).

GJ 436-GJ 436 is an M3.5 dwarf star located at a distance of $10.3 \mathrm{pc}$. It has a 45 day rotation period, a relatively old age $\left(\sim 6_{-5}^{+4}\right.$ Gyr; Torres 2007), and may have a super-solar metallicity $([\mathrm{Fe} / \mathrm{H}]=0.00-0.25$; Johnson \& Apps 2009; Rojas-Ayala et al. 2010; Neves et al. 2014). GJ 436 does show signs of an active corona with $\log _{10} L_{X}=27.16 \mathrm{erg} \mathrm{s}^{-1}$ (Poppenhaeger et al. 2010), and its chromospheric Ly $\alpha$ emission was previously observed by Ehrenreich et al. (2011) and Kulow et al. (2014). GJ 436 is notable for its well-studied transiting Neptune mass planet (Butler et al. 2004; Pont et al. 2009 ), orbiting at a semimajor axis of $\approx 0.03 \mathrm{au}$, interior to its HZ (0.16-0.31 au; von Braun et al. 2012). The impact of Ly $\alpha$ on the atmosphere of GJ 436b has been studied by Miguel et al. (2015). Additional low-mass planets may also be present in this system (Stevenson et al. 2012).

GJ $667 C-\mathrm{GJ}$ 667C (M1.5 V) is a member of a triple star system (GJ 667 AB is a $\mathrm{K} 3 \mathrm{~V}+\mathrm{K} 5 \mathrm{~V}$ binary) at a distance of 6.9 pc. This $2-10$ Gyr M dwarf (Anglada-Escudé et al. 2012) is metal-poor $([\mathrm{Fe} / \mathrm{H}]=-0.50$, Neves et al. 2014; and a similar value of $-0.59 \pm 0.10$ based on an analysis of GJ 667AB, Perrin et al. 1988). GJ 667C may host as many as three planets, including a super-Earth mass planet (GJ 667Cc, $M_{\text {plan }} \approx 4.5 M_{\oplus}, \quad a_{\text {plan }}=0.12$ au) orbiting in the $\mathrm{HZ}$ (0.11-0.23 au; Anglada-Escudé et al. 2012).

GJ $832-$ GJ 832 is an M1.5 dwarf at $d=4.9$ pc. GJ 832 is not as well characterized as other targets in our sample; an age determination for this star is not available. Coronal X-rays have been detected from GJ 832 with $\log _{10} L_{X}=26.77 \mathrm{erg} \mathrm{s}^{-1}$ (Poppenhaeger et al. 2010). This subsolar metallicity star $([\mathrm{Fe} / \mathrm{H}]=-0.17$; Neves et al. 2014) hosts two known

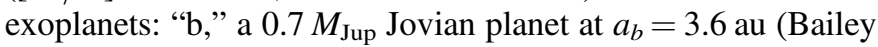
et al. 2009), and "c," a 5.4 $M_{\oplus}$ super-Earth planet in the HZ $\left(a_{c}=0.16 \mathrm{au}\right.$; Wittenmyer et al. 2014).

HD 85512-HD 85512 is a $\mathrm{K} 6$ dwarf at a distance of 11.2 pc. It is estimated to have an age of $5.6 \pm 0.1 \mathrm{Gyr}$ and a subsolar metallicity, $[\mathrm{Fe} / \mathrm{H}]=-0.26$ (Tsantaki et al. 2013) based on its 47 day rotation period. There is no previously published X-ray detection of $\mathrm{HD}$ 85512, the XMM-Newton luminosity is $\log _{10} L_{X} \sim 26.5 \mathrm{erg} \mathrm{s}^{-1}$ (Brown et al. 2016, in preparation; Loyd et al. 2016). HD 85512 b is a super-Earth mass planet $\left(3.6 M_{\oplus}\right)$ orbiting with a semimajor axis of 0.26 au (Pepe et al. 2011).
HD 40307-HD 40307 is a K2.5 dwarf at a distance of 12.9 pc. It is estimated to have an age of $4.5 \mathrm{Gyr}$ (Barnes 2007) and a subsolar metallicity, $[\mathrm{Fe} / \mathrm{H}]=-0.36$ (Tsantaki et al. 2013). HD 40307 has a 48 day rotation period (Mayor et al. 2009) and an archival X-ray luminosity of $\log _{10} L_{X}=26.99 \mathrm{erg} \mathrm{s}^{-1}$ (Poppenhaeger et al. 2010). HD 40307 hosts somewhere between 3 and 6 planets, including several that are of superEarth mass (Table 1; Mayor et al. 2009; Tuomi et al. 2013).

$\epsilon$ Eri $-\epsilon$ Eri is a K2 dwarf at a distance of $3.2 \mathrm{pc}$. It is one of the best-studied active K stars (Dring et al. 1997; Ness et al. 2002; Jeffers et al. 2014). It is a relatively young star with an age $\approx 0.44$ Gyr (Barnes 2007), an 11.68 day rotation period (Rueedi et al. 1997), and a slightly subsolar metallicity, [Fe/ $\mathrm{H}]=-0.15$ (Tsantaki et al. 2013). $\epsilon$ Eri displays magnetic cycles that suggest it may have been in a relatively "inactive" state during the MUSCLES observations (Metcalfe et al. 2013). Archival spectra from XMM-Newton show an X-ray luminosity in the $0.2-2.0 \mathrm{keV}$ energy band of $\log _{10} L_{X}=28.22 \mathrm{erg} \mathrm{s}^{-1}$ (Poppenhaeger et al. 2010) that grows to $\log _{10} L_{X}=29.32 \mathrm{erg}$ $\mathrm{s}^{-1}$ when the full range covered by the Chandra LETGS is included (0.07-2.5 keV; Ness et al. 2002). $\epsilon$ Eri hosts a $\sim 1.1 M_{\text {Jup }}$ planet in a 3.4 au semimajor axis orbit (Hatzes et al. 2000; Butler et al. 2006).

HD 97658-HD 97658 is a $\mathrm{K} 1$ dwarf at a distance of 21.1 pc. It is estimated to have an age of $3.8 \pm 2.6 \mathrm{Gyr}$ (Bonfanti et al. 2015) and a subsolar metallicity of $[\mathrm{Fe} / \mathrm{H}]=-0.26$ (Valenti \& Fischer 2005). There are no published X-ray data on HD 97658 in the literature, but X-ray observations are scheduled as part of Chandra's Cycle 16. HD 97658 hosts a super-Earth mass planet $\left(7.9 M_{\oplus}\right)$ in a short-period orbit $(a=0.08 \mathrm{au}$; Dragomir et al. 2013).

\section{REFERENCES}

Allard, F., \& Hauschildt, P. H. 1995, ApJ, 445, 433

Anglada-Escudé, G., Arriagada, P., Vogt, S. S., et al. 2012, ApJL, 751, L16 Anglada-Escudé, G., Tuomi, M., Gerlach, E., et al. 2013, A\&A, 556, A126 Ardila, D. R., Herczeg, G. J., Gregory, S. G., et al. 2013, ApJS, 207, 1 Ayres, T. R. 1997, JGR, 102, 1641

Ayres, T. R., Brown, A., Harper, G. M., et al. 2003, ApJ, 583, 963

Ayres, T., \& France, K. 2010, ApJL, 723, L38

Bailey, J., Butler, R. P., Tinney, C. G., et al. 2009, ApJ, 690, 743

Barnes, S. A. 2007, ApJ, 669, 1167

Bean, J. L., Miller-Ricci Kempton, E., \& Homeier, D. 2010, Natur, 468, 669 Berta, Z. K., Charbonneau, D., Bean, J., et al. 2011, ApJ, 736, 12

Berta-Thompson, Z. K., Irwin, J., Charbonneau, D., et al. 2015, Natur, 527, 204

Bonfanti, A., Ortolani, S., Piotto, G., \& Nascimbeni, V. 2015, A\&A, 575, A18 Bonfils, X., Delfosse, X., Udry, S., et al. 2013, A\&A, 549, A109

Brown, T. M., Baliber, N., Bianco, F. B., et al. 2013, PASP, 125, 1031 Buccino, A. P., Lemarchand, G. A., \& Mauas, P. J. D. 2007, Icar, 192, 582 Butler, R. P., Howard, A. W., Vogt, S. S., \& Wright, J. T. 2009, ApJ, 691, 1738

Butler, R. P., Vogt, S. S., Marcy, G. W., et al. 2004, ApJ, 617, 580

Butler, R. P., Wright, J. T., Marcy, G. W., et al. 2006, ApJ, 646, 505 Cauley, P. W., Redfield, S., Jensen, A. G., et al. 2015, ApJ, 810, 13

Cecchi-Pestellini, C., Ciaravella, A., Micela, G., \& Penz, T. 2009, A\&A, 496, 863

Chadney, J. M., Galand, M., Unruh, Y. C., Koskinen, T. T., \& Sanz-Forcada, J. 2015, Icar, 250, 357

Charbonneau, D., Berta, Z. K., Irwin, J., et al. 2009, Natur, 462, 891

Cincunegui, C., \& Mauas, P. J. D. 2004, A\&A, 414, 699

Correia, A. C. M., Couetdic, J., Laskar, J., et al. 2010, A\&A, 511, A21

Cowan, N. B., Greene, T., Angerhausen, D., et al. 2015, PASP, 127, 311

Demory, B.-O., Gillon, M., Barman, T., et al. 2007, A\&A, 475, 1125

Désert, J.-M., Bean, J., Miller-Ricci Kempton, E., et al. 2011, ApJL, 731, L40 Des Marais, D. J., Harwit, M. O., Jucks, K. W., et al. 2002, AsBio, 2, 153 
Domagal-Goldman, S. D., Segura, A., Claire, M. W., Robinson, T. D., \& Meadows, V. S. 2014, ApJ, 792, 90

Donahue, R. A., Saar, S. H., \& Baliunas, S. L. 1996, ApJ, 466, 384

Dragomir, D., Matthews, J. M., Eastman, J. D., et al. 2013, ApJ, 772, 2

Dressing, C. D., \& Charbonneau, D. 2015, ApJ, 807, 45

Dring, A. R., Linsky, J., Murthy, J., et al. 1997, ApJ, 488, 760

Ehrenreich, D., Lecavelier Des Etangs, A., \& Delfosse, X. 2011, A\&A, 529, A80

Endl, M., Cochran, W. D., Wittenmyer, R. A., \& Boss, A. P. 2008, ApJ, 673,1165

Engle, S. G., \& Guinan, E. F. 2011, in ASP Conf. Ser. 451, 9th Pacific Rim Conf. on Stellar Astrophysics, ed. S. Qain et al. (San Francisco, CA: ASP), 285

Fontenla, J., Witbrod, J., Linsky, J., \& France, K. 2016, ApJ, submitted

Fontenla, J. M., Harder, J., Livingston, W., Snow, M., \& Woods, T. 2011, JGRD, 116, 20108

Forveille, T., Bonfils, X., Delfosse, X., et al. 2009, A\&A, 493, 645

Fossati, L., France, K., \& Koskinen, T. 2015, ApJ, submitted

Fossati, L., Haswell, C. A., Froning, C. S., et al. 2010, ApJL, 714, L222

France, K., Froning, C. S., Linsky, J. L., et al. 2013, ApJ, 763, 149

France, K., Linsky, J. L., Tian, F., Froning, C. S., \& Roberge, A. 2012a, ApJL, 750, L32

France, K., Schindhelm, E., Bergin, E. A., Roueff, E., \& Abgrall, H. 2014, ApJ, 784, 127

France, K., Schindhelm, E., Herczeg, G. J., et al. 2012b, ApJ, 756, 171

Fressin, F., Torres, G., Charbonneau, D., et al. 2013, ApJ, 766, 81

Gaidos, E., Mann, A. W., Lépine, S., et al. 2014, MNRAS, 443, 2561

Gao, P., Hu, R., Robinson, T. D., Li, C., \& Yung, Y. L. 2015, ApJ, 806, 249

Gizis, J. E., Reid, I. N., \& Hawley, S. L. 2002, AJ, 123, 3356

Gomes da Silva, J., Santos, N. C., Bonfils, X., et al. 2011, A\&A, 534, A30

Green, J. C., Froning, C. S., Osterman, S., et al. 2012, ApJ, 744, 60

Guinan, E. F., \& Engle, S. G. 2009, eprint (arXiv:0901.1860)

Hallinan, G., Sirothia, S. K., Antonova, A., et al. 2013, ApJ, 762, 34

Harman, C. E., Schwieterman, E. W., Schottelkotte, J. C., \& Kasting, J. F. 2015, ApJ, 812, 137

Haswell, C. A., Fosatti, L. A., Ayres, T., et al. 2012, ApJ, 760, 79

Hatzes, A. P., Cochran, W. D., McArthur, B., et al. 2000, ApJL, 544, L145

Hawley, S. L., Allred, J. C., Johns-Krull, C. M., et al. 2003, ApJ, 597, 535

Hawley, S. L., Davenport, J. R. A., Kowalski, A. F., et al. 2014, ApJ, 797, 121

Hawley, S. L., \& Johns-Krull, C. M. 2003, ApJL, 588, L109

Hawley, S. L., \& Pettersen, B. R. 1991, ApJ, 378, 725

Hawley, S. L., \& Pettersen, B. R. 1991, ApJ, 10, 123

Henry, G. W., Howard, A. W., Marcy, G. W., Fischer, D. A., \& Johnson, J. A. 2011, arXiv: 1109.2549

Hu, R., Seager, S., \& Bains, W. 2012, ApJ, 761, 166

Husser, T.-O., Wende-von Berg, S., Dreizler, S., et al. 2013, A\&A, 553, A6

Jeffers, S. V., Petit, P., Marsden, S. C., et al. 2014, A\&A, 569, A79

Johnson, J. A., \& Apps, K. 2009, ApJ, 699, 933

Jones, B. W., \& Sleep, P. N. 2010, MNRAS, 407, 1259

Joshi, M. 2003, AsBio, 3, 415

Kaltenegger, L., Segura, A., \& Mohanty, S. 2011, ApJ, 733, 35

Kaltenegger, L., Traub, W. A., \& Jucks, K. W. 2007, ApJ, 658, 598

Kashyap, V. L., Drake, J. J., \& Saar, S. H. 2008, ApJ, 687, 1339

Kiraga, M., \& Stepien, K. 2007, AcA, 57, 149

Kopparapu, R. K. 2013, ApJL, 767, L8

Kopparapu, R. K., Ramirez, R. M., SchottelKotte, J., et al. 2014, ApJL, 787, L29

Kowalski, A. F., Hawley, S. L., Hilton, E. J., et al. 2009, AJ, 138, 633

Kowalski, A. F., Hawley, S. L., Holtzman, J. A., Wisniewski, J. P., \& Hilton, E. J. 2010, ApJ, 714, 98

Kreidberg, L., Bean, J. L., Désert, J.-M., et al. 2014, Natur, 505, 69

Kulow, J. R., France, K., Linsky, J., \& Loyd, R. O. P. 2014, ApJ, 786, 132

Kundurthy, P., Agol, E., Becker, A. C., et al. 2011, ApJ, 731, 123

Lai, D., Helling, C., \& van den Heuvel, E. P. J. 2010, ApJ, 721, 923

Lalitha, S., Poppenhaeger, K., Singh, K. P., Czesla, S., \& Schmitt, J. H. M. M. 2014, ApJL, 790, L11

Lammer, H., Odert, P., Leitzinger, M., et al. 2009, A\&A, 506, 399

Lanza, A. F. 2008, A\&A, 487, 1163

Lecavelier Des Etangs, A., Sirothia, S. K., Gopal-Krishna, \& Zarka, P. 2011, A\&A, 533, A50

Lecavelier des Etangs, A., Sirothia, S. K., Gopal-Krishna, \& Zarka, P. 2013, A\&A, 552, A65

Linsky, J. L., Diplas, A., Wood, B. E., et al. 1995, ApJ, 451, 335

Linsky, J. L., Fontenla, J., \& France, K. 2014, ApJ, 780, 61

Linsky, J. L., France, K., \& Ayres, T. 2013, ApJ, 766, 69

Llama, J., Wood, K., Jardine, M., et al. 2011, MNRAS, 416, L41
Loyd, R. O. P., \& France, K. 2014, ApJS, 211, 9

Loyd, R. O. P., France, K., \& Youngblood, A. 2016, ApJ, submitted

Mamajek, E. E., \& Hillenbrand, L. A. 2008, ApJ, 687, 1264

Mayor, M., Bonfils, X., Forveille, T., et al. 2009, A\&A, 507, 487

Metcalfe, T. S., Buccino, A. P., Brown, B. P., et al. 2013, ApJL, 763, L26

Miguel, Y., Kaltenegger, L., Linsky, J. L., \& Rugheimer, S. 2015, MNRAS, 446, 345

Mitra-Kraev, U., Harra, L. K., Güdel, M., et al. 2005, A\&A, 431, 679

Ness, J.-U., Schmitt, J. H. M. M., Burwitz, V., et al. 2002, A\&A, 394, 911

Neves, V., Bonfils, X., Santos, N. C., et al. 2014, A\&A, 568, A121

Oranje, B. J. 1986, A\&A, 154, 185

Osten, R. A., Hawley, S. L., Allred, J. C., Johns-Krull, C. M., \& Roark, C. 2005, ApJ, 621, 398

Owen, J. E., \& Jackson, A. P. 2012, MNRAS, 425, 2931

Pepe, F., Lovis, C., Ségransan, D., et al. 2011, A\&A, 534, A58

Perrin, M.-N., Cayrel de Strobel, G., \& Dennefeld, M. 1988, A\&A, 191, 237

Pont, F., Gilliland, R. L., Knutson, H., Holman, M., \& Charbonneau, D. 2009 MNRAS, 393, L6

Poppenhaeger, K., Robrade, J., \& Schmitt, J. H. M. M. 2010, A\&A, 515, A98

Rahmati, A., Cravens, T. E., Nagy, A. F., et al. 2014, GeoRL, 41, 4812

Rauscher, E., \& Marcy, G. W. 2006, PASP, 118, 617

Redfield, S., \& Linsky, J. L. 2002, ApJS, 139, 439

Ribas, I., Guinan, E. F., Güdel, M., \& Audard, M. 2005, ApJ, 622, 680

Rivera, E. J., Laughlin, G., Butler, R. P., et al. 2010, ApJ, 719, 890

Rivera, E. J., Lissauer, J. J., Butler, R. P., et al. 2005, ApJ, 634, 625

Robertson, P., Endl, M., Henry, G. W., et al. 2015, ApJ, 801, 79

Robertson, P., Mahadevan, S., Endl, M., \& Roy, A. 2014, Sci, 345, 440

Robinson, R. D., Linsky, J. L., Woodgate, B. E., \& Timothy, J. G. 2001, ApJ, 554, 368

Rojas-Ayala, B., Covey, K. R., Muirhead, P. S., \& Lloyd, J. P. 2010, ApJL, 720, L113

Rueedi, I., Solanki, S. K., Mathys, G., \& Saar, S. H. 1997, A\&A, 318, 429

Sanz-Forcada, J., Micela, G., Ribas, I., et al. 2011, A\&A, 532, A6

Scalo, J., Kaltenegger, L., Segura, A. G., et al. 2007, AsBio, 7, 85

Seager, S., Bains, W., \& Hu, R. 2013, ApJ, 777, 95

Seager, S., Deming, D., \& Valenti, J. A. 2009, in Astrophysics in the Next Decade (Dordrecht: Springer), 123

Seager, S., Deming, D., \& Valenti, J. A. 2009, ASSP, 10, 123

Segura, A., Kasting, J. F., Meadows, V., et al. 2005, AsBio, 5, 706

Segura, A., Walkowicz, L. M., Meadows, V., Kasting, J., \& Hawley, S. 2010, AsBio, 10, 751

Selsis, F., Kasting, J. F., Levrard, B., et al. 2007, A\&A, 476, 1373

Shkolnik, E., Bohlender, D. A., Walker, G. A. H., \& Collier, C. A. 2008, ApJ, 676,628

Shkolnik, E., Walker, G. A. H., \& Bohlender, D. A. 2003, ApJ, 597, 1092

Shkolnik, E., Walker, G. A. H., Bohlender, D. A., Gu, P.-G., \& Kürster, M. 2005, ApJ, 622, 1075

Shkolnik, E. L. 2013, ApJ, 766, 9

Shkolnik, E. L., \& Barman, T. S. 2014, AJ, 148, 64

Shkolnik, E. L., Rolph, K. A., Peacock, S., \& Barman, T. S. 2014, ApJL, 796, L20

Smith, R. K., Brickhouse, N. S., Liedahl, D. A., \& Raymond, J. C. 2001, ApJL, 556, L91

Stevenson, K. B., Harrington, J., Lust, N. B., et al. 2012, ApJ, 755, 9

Tian, F., France, K., Linsky, J. L., Mauas, P. J. D., \& Vieytes, M. C. 2014 E\&PSL, 385, 22

Tian, F., \& Ida, S. 2015, NatGe, 8, 177

Tian, F., Kasting, J. F., Liu, H.-L., \& Roble, R. G. 2008, JGRE, 113, 5008 Torres, G. 2007, ApJL, 671, L65

Tsantaki, M., Sousa, S. G., Adibekyan, V. Z., et al. 2013, A\&A, 555, A150

Tuomi, M. 2011, A\&A, 528, L5

Tuomi, M., Anglada-Escudé, G., Gerlach, E., et al. 2013, A\&A, 549, A48

Valenti, J. A., \& Fischer, D. A. 2005, ApJS, 159, 141

Vidotto, A. A., Jardine, M., \& Helling, C. 2010, ApJL, 722, L168

Vitale, V., \& France, K. 2013, A\&A, 558, A139

von Braun, K., Boyajian, T. S., Kane, S. R., et al. 2011, ApJL, 729, L26

von Braun, K., Boyajian, T. S., Kane, S. R., et al. 2012, ApJ, 753, 171

Walkowicz, L. M., \& Hawley, S. L. 2009, AJ, 137, 3297

Walkowicz, L. M., Johns-Krull, C. M., \& Hawley, S. L. 2008, ApJ, 677, 593

Welsh, B. Y., Wheatley, J., Browne, S. E., et al. 2006, A\&A, 458, 921

Welsh, B. Y., Wheatley, J. M., Seibert, M., et al. 2007, ApJS, 173, 673

Welsh, B. Y., Wheatley, J., Siegmund, O. H. W., \& Lallement, R. 2010, ApJL, 712, L199

West, A. A., Hawley, S. L., Bochanski, J. J., et al. 2008, AJ, 135, 785

Wittenmyer, R. A., Tuomi, M., Butler, R. P., et al. 2014, ApJ, 791, 114

Wood, B. E., Linsky, J. L., \& Ayres, T. R. 1997, ApJ, 478, 745 
Wood, B. E., Müller, H.-R., Zank, G. P., Linsky, J. L., \& Redfield, S. 2005a, ApJL, 628, L143

Wood, B. E., Redfield, S., Linsky, J. L., Müller, H.-R., \& Zank, G. P. 2005b, ApJS, 159, 118
Woods, T. N., Chamberlin, P. C., Harder, J. W., et al. 2009, GeoRL, 36, 1101 Wordsworth, R. D., Forget, F., Selsis, F., et al. 2010, A\&A, 522, A22

Wordsworth, R. D., Forget, F., Selsis, F., et al. 2011, ApJL, 733, L48

Youngblood, A., France, K., \& Loyd, R. O. P. 2016, ApJ, submitted 